\title{
Novel flavour-changing neutral currents in the top quark sector
}

\author{
Nuno Castro, ${ }^{a}$ Mikael Chala, ${ }^{b}$ Ana Peixoto ${ }^{a}$ and Maria Ramos ${ }^{a}$ \\ ${ }^{a}$ Laboratório de Instrumentação e Física Experimental de Partículas, \\ Departamento de Física, Universidade do Minho, \\ 4710-057 Braga, Portugal \\ ${ }^{b}$ CAFPE and Departamento de Física Teórica y del Cosmos, Universidad de Granada, \\ E-18071 Granada, Spain \\ E-mail: nuno.castro@fisica.uminho.pt, mikael.chala@ugr.es, \\ apeixoto@lip.pt, mariaramos@lip.pt
}

ABSTRACT: We demonstrate that flavour-changing neutral currents in the top sector, mediated by leptophilic scalars at the electroweak scale, can easily arise in scenarios of new physics, and in particular in composite Higgs models. We moreover show that such interactions are poorly constrained by current experiments, while they can be searched for at the LHC in rare top decays and, more generally, in the channels $p p \rightarrow t S(S)+j$, with $S \rightarrow \ell^{+} \ell^{-}$. We provide dedicated analyses in this respect, obtaining that cut-off scales as large as $\Lambda \sim 90 \mathrm{TeV}$ can be probed with an integrated luminosity of $\mathcal{L}=150 \mathrm{fb}^{-1}$.

Keywords: Beyond Standard Model, Flavour Changing Neutral Currents, Hadron-Hadron scattering (experiments), Top physics

ARXIV EPRINT: 2005.09594 


\section{Contents}

1 Introduction 1

2 Interactions and constraints 2

3 Search for $t \rightarrow S q, S \rightarrow \mu^{+} \mu^{-} \quad 6$

4 Search for $t \rightarrow S q, S \rightarrow \tau^{+} \tau^{-} \quad 9$

5 Search for $t \rightarrow S S q, S \rightarrow \mu^{+} \mu^{-} \quad 13$

$\begin{array}{lll}6 & \text { Conclusions } & 17\end{array}$

\section{Introduction}

New pseudoscalars $S$ with mass $m_{S}$ close to the electroweak (EW) scale, singlets of the Standard Model (SM) gauge group, have two opposing faces. On one hand, they are predicted in very different and well motivated scenarios of new physics. These include, among others, the NMSSM [1] in which an extra supersinglet reduces the $\mu$-problem; as well as a big part of the composite Higgs models (CHM) developed to the date [2-15]. Moreover, pseudoscalar singlets have been showed to be excellent candidates to accommodate EW baryogenesis with two step phase transitions at which CP is spontaneously broken [10, 15-21]. Also, they have been proved to explain the $g-2$ anomaly of the muon [22].

However, on the other hand, pseudoscalar singlets around the EW scale are very difficult to detect. The first reason is that at the renormalisable level they only interact with the Higgs boson. If they are above the threshold $\sim m_{h} / 2$, they are therefore produced only with extremely low cross section by means of an off-shell Higgs; being even out of the reach of a potential $100 \mathrm{TeV}$ collider [23, 24]. Moreover, the strong constraints on dipole moments $[25,26]$ forbids any sizable mixing with the Higgs, while other production mechanisms are mediated by higher-dimensional operators and therefore suppressed by the cut-off scale $[27,28]$, that hereafter we refer to as $\Lambda$.

In light of these results, there has been research exploring novel production mechanisms for new pseudoscalar singlets. One of the most exciting possibilities is producing such particles in the decay of top quarks via effective interactions. Such proposal aims to exploit the huge top quark production rate at the Large Hadron Collider (LHC) and future facilities. Search strategies for $t \rightarrow S q, S \rightarrow b \bar{b}(\gamma \gamma)$ have been discussed in ref. [29]. (See refs. [30-32] for studies focused on $m_{S} \sim m_{h} \sim 125 \mathrm{GeV}$ and refs. [33, 34] for experimental works.) For $m_{S} \gtrsim m_{t} \sim 172 \mathrm{GeV}$, the top quark decays non resonantly; being adequately described by four-fermion interactions [35]. 
In this article, we extend previous works in this topic in three ways. First, we consider the rare top decay $t \rightarrow S q, S \rightarrow \ell^{+} \ell^{-}$including both light leptons and taus. Second, we include the effect of the flavour-violating vertices not only in the decay, but also in the production of top quarks. ${ }^{1}$ And third, we demonstrate that a more natural new top decay is $t \rightarrow S S, S \rightarrow \ell^{+} \ell^{-}$and we also study in detail the LHC reach to this process.

The text is organized as follows. In section 2 we introduce the relevant dimensionsix effective field theory Lagrangian of the SM extended with $S$ and compare to concrete models. We show which interactions are already constrained by current Higgs and flavour data, and define several benchmark points (BP) for the subsequent study of top decays. We dedicate sections 3 and 4 to explore the collider phenomenology of $t \rightarrow S q$ with $S \rightarrow \ell^{+} \ell^{-}$ and $S \rightarrow \tau^{+} \tau^{-}$, respectively. In section 5 we concentrate on $t \rightarrow S S q, S \rightarrow \ell^{+} \ell^{-}$. We conclude in section 6 .

\section{Interactions and constraints}

The most generic Lagrangian describing the interactions (that can be induced at tree level in UV completions of the SM to dimension six) between a scalar singlet $S$ with mass $m_{S}$ and the SM fields reads [27]:

$$
\begin{aligned}
\Delta L= & -\frac{1}{2} \lambda_{H S} S^{2}\left(|H|^{2}-\frac{v^{2}}{2}\right)+c_{H S} \frac{(\partial S)^{2}}{\Lambda^{2}}|H|^{2} \\
& +\frac{S}{\Lambda} \overline{\mathbf{f}_{\mathbf{L}}} \mathbf{Y}^{\mathbf{f}} H \mathbf{f}_{\mathbf{R}}+\frac{S^{2}}{\Lambda^{2}}\left[c_{H S}^{\prime}|D H|^{2}+\tilde{c}_{H S}\left(|H|^{4}-\frac{v^{4}}{4}\right)+\overline{\mathbf{f}_{\mathbf{L}}} \tilde{\mathbf{Y}}^{\mathbf{f}} H \mathbf{f}_{\mathbf{R}}\right] .
\end{aligned}
$$

(The addition of the hermitian conjugate in the fermions, as well as $\tilde{H}=i \sigma_{2} H^{*}$ when needed, is implied; with $H$ being the Higgs doublet and $\sigma_{2}$ the second Pauli matrix.) We note that $f$ runs over quarks and leptons, $f=q, l$. Let us work in the approximation that the Cabibbo-Kobayashi-Maskawa matrix is fully diagonal, then no rotation is needed on $\mathbf{f}_{\mathbf{L}, \mathbf{R}}$. The different Wilson coefficients in the expression above are subject to a number of constraints. Thus, $\lambda_{H S}, c_{H S}$ and $\tilde{c}_{H S}$ enter the Higgs width:

$$
\Gamma(h \rightarrow S S)=\frac{v^{2}}{32 \pi m_{h}} \sqrt{1-\frac{4 m_{S}^{2}}{m_{h}^{2}}}\left[\lambda_{H S}+c_{H S} \frac{\left(m_{h}^{2}-2 m_{S}^{2}\right)}{\Lambda^{2}}-2 \tilde{c}_{H S} \frac{v^{2}}{\Lambda^{2}}\right]^{2} .
$$

Therefore, values of $m_{S}<m_{h} / 2$ are a priori constrained by LHC measurements on the Higgs width, $\Gamma_{H} \lesssim 10 \mathrm{MeV}$ [37]. This bound can be evaded only if cancellations between the different operators in the bracket make this smaller than $\sim 0.05$. Assuming one operator at a time, we obtain the bounds $\lambda_{H S} \lesssim 0.05 ; c_{H S} / \Lambda^{2} \lesssim 3(5) \mathrm{TeV}^{-2}$ for $m_{S}=10(50) \mathrm{GeV}$; and $-\tilde{c}_{H S} / \Lambda^{2} \lesssim 0.4 \mathrm{TeV}^{-2}$.

Entries in $\mathbf{Y}^{\mathbf{f}}$ and $\tilde{\mathbf{Y}}^{\mathbf{f}}$ are constrained by e.g. direct searches for resonances [38, 39]. One important exception is entries $i 3,3 i$ of $\mathbf{Y}^{\mathbf{q}}$. There are no direct limits on these. Moreover, indirect constraints from flavour experiments, e.g. $D^{0}-\bar{D}^{0}$ oscillations [40-42], involve always products of two different Yukawas. They are therefore negligible if e.g. the

\footnotetext{
${ }^{1}$ The interference effects between the production and decay modes were shown to be negligible in ref. [36].
} 
entry 13 or 23 of $\mathbf{Y}^{\mathbf{q}}$ vanishes [29]; same for $\tilde{\mathbf{Y}}^{\mathbf{q}}$. However, they can be observable in new experiments. Indeed, after EWSB and for $m_{t}>m_{S}\left(2 m_{S}\right)$, they lead to signatures such as $t \rightarrow q S(S)$ arising from

$$
\Delta L \supset \frac{v S}{\sqrt{2} \Lambda}\left[\mathbf{Y}_{i 3}^{\mathbf{q}} \overline{u_{L}^{i}} t_{R}+\mathbf{Y}_{3 i}^{\mathbf{q}} \overline{t_{L}} u_{R}^{i}+\frac{S}{\Lambda}\left(\tilde{\mathbf{Y}}_{i 3}^{\mathbf{q}} \overline{u_{L}^{i}} t_{R}+\tilde{\mathbf{Y}}_{3 i}^{\mathbf{q}} \overline{t_{L}} u_{R}^{i}\right)+\text { h.c. }\right]
$$

with $i=1,2$.

The decay widths read respectively:

$$
\begin{aligned}
\Gamma\left(t \rightarrow q^{i} S\right)=\frac{v^{2}}{64 \pi \Lambda^{2}}\left[\left(\mathbf{Y}_{i 3}^{\mathbf{q}}\right)^{2}+\left(\mathbf{Y}_{3 i}^{\mathbf{q}}\right)^{2}\right] m_{t}\left(1-x^{2}\right)^{2} \\
\Gamma\left(t \rightarrow q^{i} S S\right)=\frac{v^{2}}{512 \pi^{3} \Lambda^{4}}\left[\left(\tilde{\mathbf{Y}}_{i 3}^{\mathbf{q}}\right)^{2}+\left(\tilde{\mathbf{Y}}_{3 i}^{\mathbf{q}}\right)^{2}\right] m_{t}^{3}\left[\frac{1}{3} \sqrt{1-4 x^{2}}\left(1+5 x^{2}-6 x^{4}\right)\right. \\
\left.+2\left(x^{2}-2 x^{4}+2 x^{6}\right) \log \frac{2 x^{2}}{1-2 x^{2}+\sqrt{1-4 x^{2}}}\right]
\end{aligned}
$$

where we have defined $x=m_{S} / m_{t}$. Taking $\Gamma_{t} \sim 1.4 \mathrm{GeV}$ as reference value of the top width [43], we show in the left panel of figure 1 the branching ratio of the top quark into $S q$ and $S S q$ for $\tilde{\mathbf{Y}}_{i 3}^{\mathbf{q}}=\widetilde{\mathbf{Y}}_{3 i}^{\mathbf{q}}=\mathbf{Y}^{\mathbf{q}}{ }_{i 3}=\mathbf{Y}^{\mathbf{q}}{ }_{3 i}=1$ and $\Lambda=1 \mathrm{TeV}$ for different values of $m_{S}$.

The scalar $S$ can subsequently decay into fermions. In this article we focus on the channel $S \rightarrow \ell^{+} \ell^{-}$. Assuming that this decay mode dominates the $S$ width while $t \rightarrow q^{i} S$ requires that only $\mathbf{Y}^{\mathbf{q}_{i 3}}$ (and/or $\mathbf{Y}_{3 i}^{\mathbf{q}_{3}}$ ) and $\mathbf{Y}_{j j}^{\mathbf{l}}, j=1,2,3$, are non vanishing. This scenario does not easily arise in UV models, where diagonal couplings of $S$ to quarks are generally also present, proportional to masses, and $b \bar{b}$ dominates the $S$ width; also due to the larger number of colours with respect to leptons [29]. Still, the branching ratio to taus is only an order of magnitude smaller. Thus, we will consider this unexplored $S$ decay in the context of top flavor-changing neutral currents (FCNCs) in this paper. For its cleanness, we will also consider the dimuon channel.

Prospects are very different if $t \rightarrow q^{i} S S$ instead. From the theory point of view, it can well be that a $\mathbb{Z}_{2}$ symmetry $S \rightarrow-S$ is only (or mostly) broken in the lepton sector. Or even just in the muon and electron side; in which case the dilepton decay of $S$ is dominant. Let us write $\mathbf{Y}^{\mathbf{f}}{ }_{j j}=\gamma_{f_{j}} y_{f_{j}}$, where $y_{f_{j}}$ is the fermion Yukawa and $0<\gamma_{f_{j}}<1$ parameterizes the degree of breaking of the $\mathbb{Z}_{2}$. In the right panel of figure 1 we show the branching ratio of $S$ into taus and muons for different assumptions on this parameter.

The interactions above arise very naturally within CHMs $[44,45]$, where both the Higgs and $S$ are pseudo-Goldstone bosons associated with the spontaneous global symmetry breaking $\mathcal{G} / \mathcal{H}$ driven in a new strong sector at the confinement scale $\Lambda \sim \mathrm{TeV}$. In these models, the global symmetry is only approximate; being explicitly broken by the linear mixing between the elementary SM fermions and composite operators. (Or equivalently, by embedding the SM fermions in incomplete multiplets of $\mathcal{G}$.)

As a matter of example, let us consider the next-to-minimal CHM based on the coset $\mathrm{SO}(6) / \mathrm{SO}(5)$ [3]. The generators of $\mathrm{SO}(6)$ can be split into $\mathrm{SO}(5)$ generators, $T$, and coset 

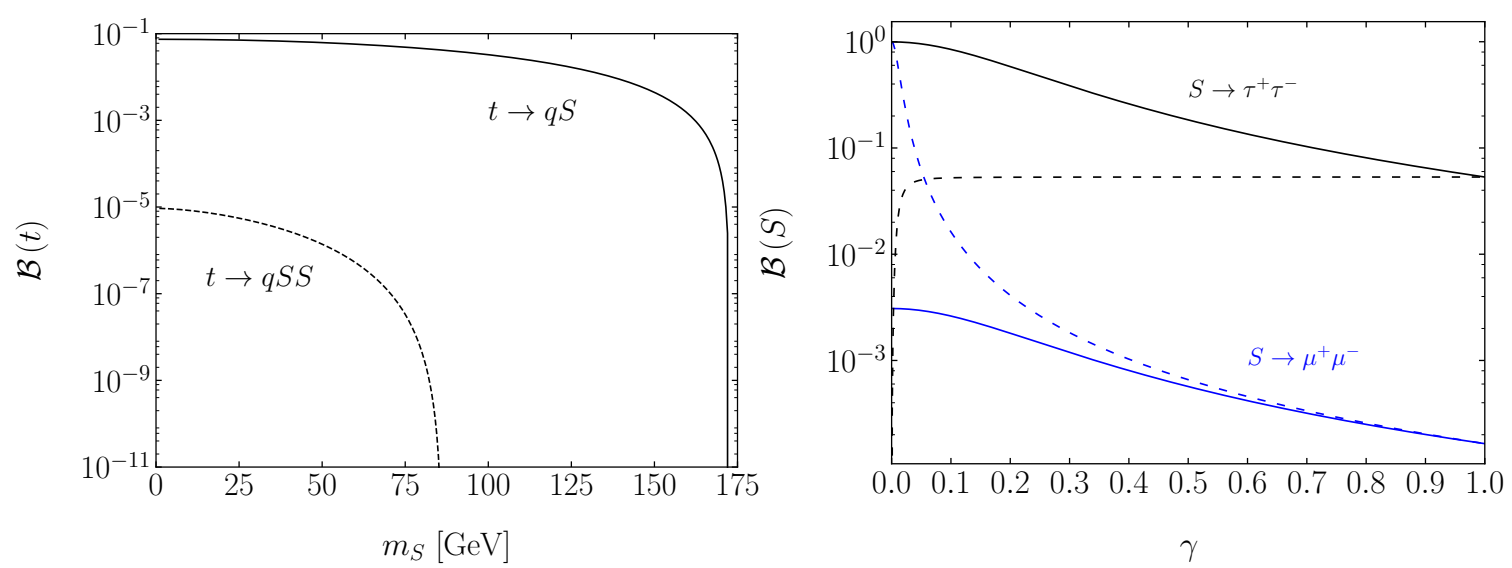

Figure 1. Left) Top branching ratios as a function of the mass of $S$, for $\tilde{\mathbf{Y}}_{i 3}^{\mathbf{q}}=\widetilde{\mathbf{Y}}_{3 i}^{\mathbf{q}}=\mathbf{Y}^{\mathbf{q}}{ }_{i 3}=$ $\mathbf{Y}^{\mathbf{q}}{ }_{3 i}=1$ and $\Lambda=1 \mathrm{TeV}$. Right) Scalar branching ratios into muons (blue) and taus (black), as a function of the $\mathbb{Z}_{2}$ breaking parameter, $\gamma$, for $m_{S}=100 \mathrm{GeV}$. We represent two cases: (in solid lines) $\gamma_{\ell}=\gamma_{\tau}=1$ while $\gamma_{q}=\gamma$; and (in dashed lines) $\gamma_{\ell}=1$ and $\gamma_{\tau}=\gamma_{q}=\gamma$.

generators, $X$ :

$$
T_{i j}^{m n}=-\frac{i}{\sqrt{2}}\left(\delta_{i}^{m} \delta_{j}^{n}-\delta_{i}^{n} \delta_{j}^{m}\right) \quad X_{i j}^{m n}=-\frac{i}{\sqrt{2}}\left(\delta_{i}^{m} \delta_{j}^{6}-\delta_{i}^{6} \delta_{j}^{m}\right),
$$

with $m<n \in[1,5]$. The pNGB matrix reads

$$
\mathrm{U}=\left[\begin{array}{ccc}
1_{3 \times 3} & & \\
1-h^{2} /\left(\Lambda^{2}+\Pi\right) & -h S /\left(\Lambda^{2}+\Pi\right) & h / \Lambda \\
-h S /\left(\Lambda^{2}+\Pi\right) & 1-S^{2} /\left(\Lambda^{2}+\Pi\right) & S / \Lambda \\
-h / \Lambda & -S / \Lambda & \Pi / \Lambda^{2}
\end{array}\right], \quad \Pi=\Lambda^{2}\left(1-\frac{h^{2}}{\Lambda^{2}}-\frac{S^{2}}{\Lambda^{2}}\right)^{1 / 2}
$$

Let us consider the regime in which the left-handed quarks are embedded in the representation $\mathbf{6}$, while right-handed up quarks do in both the $\mathbf{6}$ and the 15. Explicitly:

$$
\begin{aligned}
Q_{L}^{I}=\frac{1}{\sqrt{2}}\left(i d_{L}^{I}, d_{L}^{I}, i u_{L}^{I},-u_{L}^{I}, 0,0\right), & U_{R_{1}}^{I}=\left(0,0,0,0, i \gamma_{q} u_{R}^{I}, u_{R}^{I}\right) \\
\text { and } \quad & U_{R_{2}}^{I}=i\left(T^{12}-T^{34}\right) u_{R}^{I},
\end{aligned}
$$

with $I$ running over the three quark families and $\gamma_{q}$ being a positive number. To zero momentum and two fermions, only two invariants can be built upon the spurions above. One arises from the product of the singlets in the decompositions $\mathbf{6}_{Q_{L}, U_{R_{1}}}=\mathbf{1}+\mathbf{5}$. The second one results from the scalar product of the fiveplets in the decompositions $\mathbf{6}_{Q_{L}}=\mathbf{1}+\mathbf{5}$ and $15_{U_{R_{2}}}=5+\mathbf{1 0}$. Mathematically:

$$
\begin{aligned}
L & =\Lambda y_{I J}^{(1)}\left(\overline{U^{T} Q_{L}^{I}}\right)_{6}\left(U^{T} U_{R_{1}}^{J}\right)_{6}-\Lambda y_{I J}^{(2)}\left(\overline{U^{T} Q_{L}^{I}}\right)_{m}\left(\operatorname{Tr}\left[U^{T} U_{R_{2}}^{J} U X^{m 6}\right]\right) \\
& =\frac{1}{\sqrt{2}} \overline{u_{L}^{I}} h u_{R}^{J}\left[y_{I J}^{(1)}\left(-1+i \gamma_{q} \frac{S}{\Lambda}+\frac{h^{2}}{2 \Lambda^{2}}+\frac{S^{2}}{2 \Lambda^{2}}\right)+y_{I J}^{(2)}+\cdots\right]+\text { h.c. },
\end{aligned}
$$


with the ellipsis representing terms suppressed by further powers of $1 / \Lambda$. Comparing the two equations above with eq. (2.3), we find that $\mathbf{Y}^{\mathbf{q}}{ }_{i j} \sim \gamma_{q} y_{i j}^{(1)}$ and $\tilde{\mathbf{Y}}_{i j}^{\mathbf{q}} \sim y_{i j}^{(1)}$. Thus, in general, these matrices are not aligned with the Yukawa matrix $\sim y^{(1)}-y^{(2)}$ and therefore introduce FCNCs.

If the leptons are only embedded in six-dimensional representations we obtain, upon rotation:

$$
L=-\frac{y_{l}^{I}}{\sqrt{2}} \overline{l_{L}^{I}} h e_{R}^{I}\left[1-\gamma_{l} \frac{S}{\Lambda}+\cdots\right]+\text { h.c. } .
$$

The assumption that leptons mix with only one representation of the composite sector implies that FCNCs vanish; $\mathbf{Y}^{\mathbf{l}}$ is automatically diagonal in the physical basis. In fact it is proportional to the lepton Yukawa matrix. Thus, the $S$ decay to taus is expected to dominate. $S \rightarrow \mu^{+} \mu^{-}$should not be neglected, though. First, because $m_{\mu} / m_{\tau} \sim 0.06$ is not dramatically small. And second because if taus couple only to the $\mathbf{1 5}$, or $\gamma_{\tau}$ is small, then the muon channel dominates the $S$ width; ${ }^{2}$ we refer again to figure 1.

We also note that, within the class of CHMs we have just described, there are also Higgs mediated FCNCs. Searches for these have been performed in e.g. refs. [33, 34]. However, as it was first pointed out in ref. [29], exploring $S$ mediated FCNCs is much more promising for several reasons: (i) they arise at dimension five, and therefore are less suppressed by powers of $v / \Lambda$; (ii) the mass of $S$ can lie at values where the SM background is less prominent; and (iii) contrary to what occurs in the case of $S$, there is no parameter space in which the Higgs boson can decay sizably into the cleanest final states such as $\mu^{+} \mu^{-}$.

Currently, only a few experimental searches are (marginally) sensitive to the interactions discussed before. The first one is the ATLAS search for $t \rightarrow Z q$ of ref. [46]. In the control region dubbed CR1, this analysis requires three light leptons (either electrons or muons, denoted by $\ell$ ), two of them of the same flavour and opposite sign (SFOS), exactly one $b$-tagged jet and at least two more light jets. Most importantly, the two SFOS leptons with invariant mass closer to the $Z$ mass $\sim 91.2 \mathrm{GeV}$ are required to be out of a $15 \mathrm{GeV}$ mass window around the $Z$ pole. Consequently $t \rightarrow S q$ events with $m_{S} \neq m_{Z}$ are captured in this region.

The possibility of using this control region to constrain interactions not necessarily leading to $t \rightarrow Z q$ was first pointed out in ref. [35], which also reports the maximum number of signal events allowed by the analysis to be $s_{\max }=143$. In order to estimate the LHC sensitivity to the proposed signal, we rely on home-made routines based on ROOT v6 [47] with Fast Jet v3 [48]. The simulated events were generated with MadGraph v5 [49] and Pythia v8 [50]. For the efficiency for selecting $t \bar{t}$ events in the semileptonic channel we obtain $\epsilon \sim 0.2$. The expected number of signal events at $\mathcal{L}=36 \mathrm{fb}^{-1}$ reads therefore

$$
N \sim 2 \times \sigma(p p \rightarrow t \bar{t}) \times \mathcal{B}\left(t \rightarrow \ell^{+} \nu b\right) \times \mathcal{B}\left(t \rightarrow S q, S \rightarrow \ell^{+} \ell^{-}\right) \times \mathcal{L} \times \epsilon,
$$

with $\mathcal{B}\left(t \rightarrow \ell^{+} \nu b\right) \sim 0.27$ [43] and $\sigma(p p \rightarrow t \bar{t})=832 \pm 29 \mathrm{pb}$ at $13 \mathrm{TeV}$ [51]. This implies that the upper limit on $\mathcal{B}\left(t \rightarrow S q, S \rightarrow \ell^{+} \ell^{-}\right)$is $\sim 143 /\left(3 \times 10^{6}\right) \sim 5 \times 10^{-5}$. This bound

\footnotetext{
${ }^{2}$ We remark that, in both scenarios with $\gamma_{\tau} \rightarrow 1$ or $\gamma_{\tau} \rightarrow 0$ and $\gamma_{\mu} \rightarrow 1$, the flight distance of $S$ is about $10^{-9}-10^{-6} \mathrm{~cm}$. Therefore, the singlet decays promptly.
} 
in turn translates to a bound on $\left(\mathbf{Y}^{\mathbf{q}} i 3\right)^{2}+\left(\mathbf{Y}^{\mathbf{q}_{3 i}}\right)^{2} \lesssim 10^{-4}(\Lambda / v)^{2}$, (for $\left.m_{S} \sim m_{t} / 2\right)$. To the best of our knowledge there are no relevant constraints on the tau channel.

Another analysis sensitive to the proposed top interactions is the ATLAS search for SUSY in multilepton final states [52]. The maximum number of allowed signal events in this analysis for $L=150 \mathrm{fb}^{-1}$ is larger than 20. Upon implementing only a few of the cuts, we have checked that such big number arises within our framework only if FCNC couplings are larger than 1 for $\Lambda=1 \mathrm{TeV}$. It will become clear in the following sections that the dedicated analyses we propose are sensitive to a much larger region of the parameter space.

Other similar searches suffer from the same problem. Namely, they are too broad in scope and therefore the background is large enough to hide the signal we are interested in. Consequently, dedicated searches are required and we discuss three examples in very detail in the subsequent sections, aiming to explore $p p \rightarrow t S+j, S \rightarrow \mu^{+} \mu^{-} ; p p \rightarrow t S+j$, $S \rightarrow \tau^{+} \tau^{-}$and $p p \rightarrow t S S+j, S \rightarrow \mu^{+} \mu^{-}$. Note hence that the top FCNCs can be either in the decay of the top quark when is pair-produced via QCD (with the extra jet from radiation), or directly in the core of $t S$ associate production.

In light of the discussion above, we assume hereafter that $\Lambda \gtrsim \mathrm{TeV}$ and all Wilson coefficients vanish with the exception of ${ }^{3} \mathbf{Y}_{22}^{\mathbf{l}}$ (or $\mathbf{Y}_{33}^{l}$ ) and either $\mathbf{Y}_{13}^{\mathbf{q}}$ or $\mathbf{Y}_{23}^{\mathbf{q}}$ or $\tilde{\mathbf{Y}}_{13}^{\mathbf{q}}$ (depending on which process we study). We quantify the results in terms of seven benchmark masses: $m_{S}=20,50,80,90,100,120$ and $150 \mathrm{GeV}$. Given the reduced phase space for $t \rightarrow S S q$, we include in addition the benchmark masses $m_{S}=30,40,60$ and $70 \mathrm{GeV}$ in the analysis for this channel. The reach of the dedicated analyses proposed below will be compared to the following Benchmark Points (BP):

$$
\begin{array}{lclll}
\text { BP 1: } & \mathbf{Y}^{\mathbf{q}}{ }_{i 3}=\mathbf{Y}^{\mathbf{q}}{ }_{3 i}=0.01, & \Lambda=5 \mathrm{TeV} & \Longrightarrow & \mathcal{B}(t \rightarrow S q) \sim 10^{-8}-10^{-7}, \\
\text { BP 2: } & \mathbf{Y}_{i 3}^{\mathbf{q}}=\mathbf{Y}_{3 i}=0.10, & \Lambda=5 \mathrm{TeV} & \Longrightarrow & \mathcal{B}(t \rightarrow S q) \sim 10^{-6}-10^{-5}, \\
\text { BP 3: } & \mathbf{Y}^{\mathbf{q}}{ }_{i 3}=\mathbf{Y}_{3 i}=0.10, & \Lambda=1 \mathrm{TeV} & \Longrightarrow & \mathcal{B}(t \rightarrow S q) \sim 10^{-4}-10^{-3}, \\
\text { BP 4: } & \tilde{\mathbf{Y}}_{i 3}^{\mathbf{q}}=\tilde{\mathbf{Y}}_{3 i}^{\mathbf{q}}=1.00, & \Lambda=5 \mathrm{TeV} & \Longrightarrow & \mathcal{B}(t \rightarrow S S q) \sim 10^{-11}-10^{-8}, \\
\text { BP 5: } & \tilde{\mathbf{Y}}_{i 3}^{\mathbf{q}}=\tilde{\mathbf{Y}}_{3 i}^{\mathbf{q}}=0.20, & \Lambda=1 \mathrm{TeV} & \Longrightarrow & \mathcal{B}(t \rightarrow S S q) \sim 10^{-10}-10^{-7}, \\
\text { BP 6: } & \tilde{\mathbf{Y}}_{i 3}^{\mathbf{q}}=\tilde{\mathbf{Y}}_{3 i}^{\mathbf{q}}=1.00, & \Lambda=1 \mathrm{TeV} & \Longrightarrow & \mathcal{B}(t \rightarrow S S q) \sim 10^{-8}-10^{-5},
\end{array}
$$

with $i=1,2$. The range in the branching ratio ensues from the range of values of $m_{S}$.

\section{Search for $t \rightarrow S q, S \rightarrow \mu^{+} \mu^{-}$}

The singlet $S$ can arise either in the production or in the decay of the top quark; see the diagrams on figure 2 . This leads to a final state with exactly one $S$, one top quark decaying into $W b$ and eventually an additional light quark $q=u, c$. In this first analysis we study the scenario where $S$ decays into a pair of muons. We focus on the leptonic decay of the $W$. Hence, at the detector level, we expect three charged leptons, several jets (at least one originated by a $b$ meson) and significant missing energy.

\footnotetext{
${ }^{3}$ Note that for $10 \mathrm{GeV}<m_{S}<100 \mathrm{GeV}$ current data from ATLAS, CMS and BaBar only constrain $\mathbf{Y}^{\mathbf{l}} \gtrsim 0.1$ [22] for $\Lambda=1 \mathrm{TeV}$. Even for much smaller values, $S$ decays promptly. We remark that LEP bounds at the $Z$ pole [53] are more than one order of magnitude weaker than the previous constraints.
} 


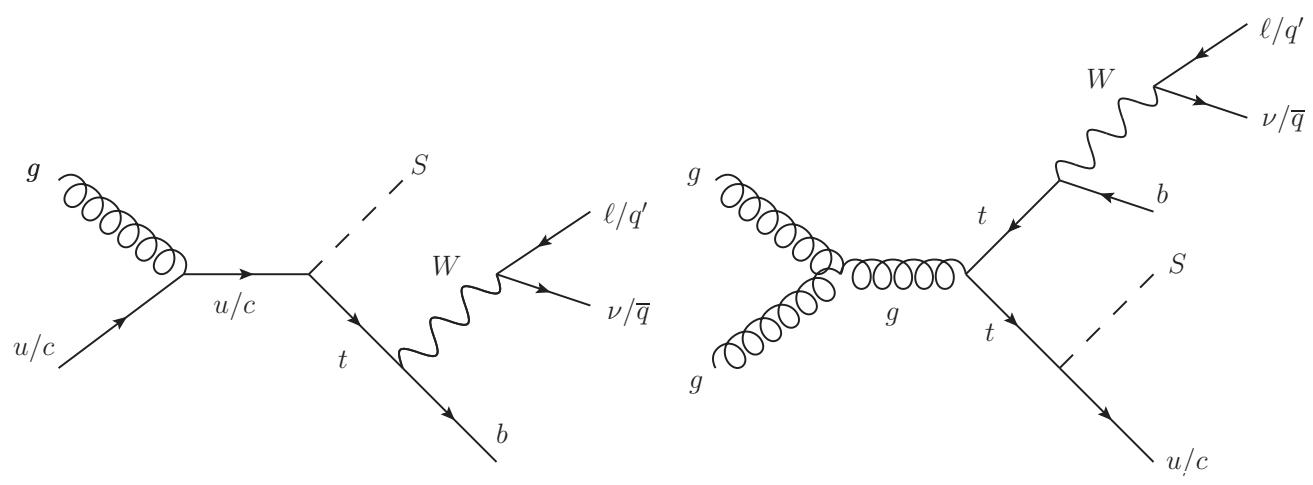

Figure 2. Representative Feynman diagrams for the production of a single top quark in association with $S$ via an FCNC interaction (left) and for the top-quark pair production with an FCNC topquark decay into the extra singlet (right). Similar diagrams but involving two rather than one $S$ in the new physics vertex hold for the production of a top quark in association with $S S$.

We generate signal and background events at $\sqrt{s}=13 \mathrm{TeV}$ with MadGraph v5 [49], with signal model being implemented in Feynrules v2 [54]. We subsequently use Pythia v8 [50] for simulating the initial and final state radiation, the parton shower and the hadronization. At parton level, only leptons and photons with a transverse momentum higher than $10 \mathrm{GeV}$ are considered. For the jets, this cut rises to $20 \mathrm{GeV}$. Concerning the absolute pseudo-rapidity $|\eta|$, jets can have a value of this variable lower than 5 while for leptons and photons it should be lower than 2.5.

We use the Parton Distribution Functions NNPDF23LO [55] and set the renormalization and factorization scales to the default dynamical MadGraph value. The total background comprises samples from $t W, t \bar{t} V, V V, Z V V, t \bar{t}, V+$ jets and $t Z$, with $V=W, Z$. For the present analysis, the most dominant background comes from $t Z$ and $t \bar{t}$ production, where $t \rightarrow W b$ and all gauge bosons are assumed to decay into muons. Such exclusive samples are motivated by the targeted trilepton final state of the signal. (Although the $t W$, diboson and $Z+$ jets processes have the largest cross sections, they become irrelevant after the cuts on the number of reconstructed leptons and jets; check table 3.)

We use Delphes [56] to simulate the detector effects with the default CMS detector card. An electron (muon) is considered to be isolated if the sum of the transverse momenta of all particles above $p_{T}^{\min }=0.5 \mathrm{GeV}$ that lie within a cone of radius $R=0.5$, normalized to the lepton $p_{T}$, is smaller than $0.12(0.25)$.

Jets are defined using the anti- $k_{t}$ algorithm [57] with a radius parameter of $R=0.5$. All the jets are required to have $p_{T}>25 \mathrm{GeV}$ and to lie within a pseudorapidity range of $|\eta|<2.5$. Leptons must have $p_{T}>15 \mathrm{GeV}$ and $|\eta|<2.4$ (2.5) for muons (electrons); the hardest lepton is also required to have $p_{T}>25 \mathrm{GeV}$. The effect of the previous cuts on the transverse momentum and pseudorapidity, together with the requirement of exactly three isolated leptons, can be found in the yields tables labeled as "basic".

We then select events with at least one jet, one of them required to be tagged as a $b$-quark. The Delphes CMS card was used to parameterize the $p_{T}$ dependent tagging efficiencies for jets initiated by $b$-quarks, as well as to take into account the mistag probability. 

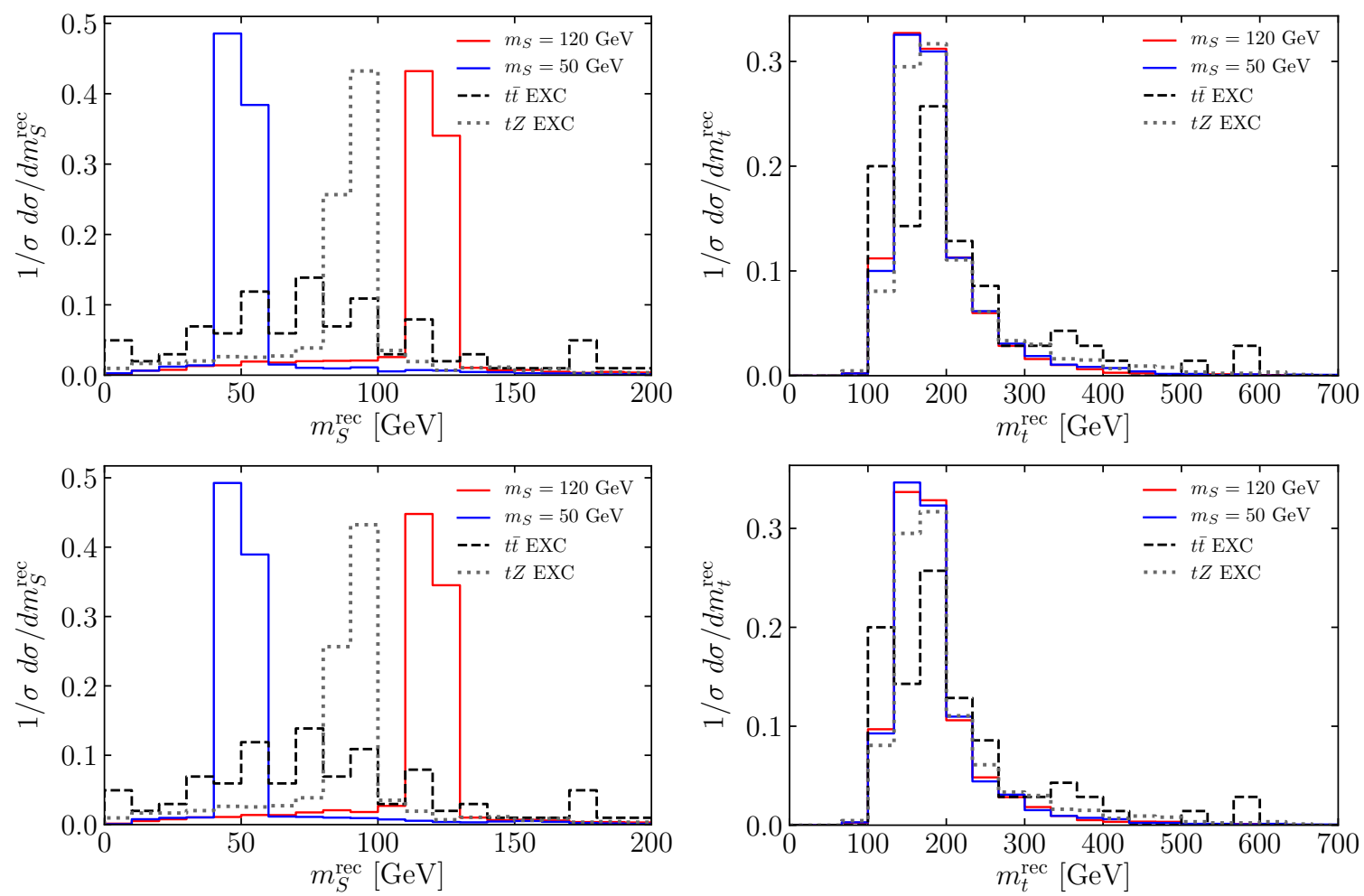

Figure 3. The reconstructed scalar (left) and top (right) mass in the analysis proposed for $t \rightarrow S q$, $S \rightarrow \mu^{+} \mu^{-}$. In the upper (bottom) panel, $q=c(u)$. We represent the distributions of two signal benchmark points and the two major background components, after the cut on the particle multiplicities; the background samples are generated exclusively, i.e. only gauge boson decays into muons are included. The distributions assume a collected luminosity of $\mathcal{L}=150 \mathrm{fb}^{-1}$.

As an example, for a $b$-jet with a transverse momentum of $30 \mathrm{GeV}$, the tagging efficiency is $55 \%$ and the mistag rate for a $c$-jet with the same $p_{T}$ is $12 \%$. The scalar resonance, $S$, is reconstructed from the hardest $\mu^{+} \mu^{-}$pair (if there is none, the event is discarded). The longitudinal component of the missing neutrino four-momentum $\left(p_{\nu}\right)$ is reconstructed by demanding $m_{W}^{2}=\left(p_{\ell}+p_{\nu}\right)^{2}$, where $p_{\ell}$ is the four-momentum of the lepton not coming from the scalar decay and $m_{W}=81.2 \mathrm{GeV}$ is our reference value for the $W$ boson mass. Among the two possible solutions, we use the one with smaller absolute value.

Both $p_{\nu}$ and $p_{\ell}$ are then added to the four-momentum of the $b$-jet to reconstruct the SM top quark; its invariant mass being dubbed $m_{t}^{\text {rec }}$. We show the distributions of the scalar and top reconstructed masses for two signal benchmark points and for the relevant background components in figure 3. The label EXC manifests that the corresponding backgrounds are generated assuming the exclusive leptonic mode. We require the $m_{t, \text { rec }}$ variable to be within a window of $50 \mathrm{GeV}$ from the reference top mass $m_{t}=172.5 \mathrm{GeV}$. We impose an additional cut of $1 \mathrm{TeV}$ on the maximum invariant mass of the total system, $m_{\text {total }}$, in order to stay in the regime of validity of the effective field theory. The impact on the expected signal yield caused by this additional requirement is minor when compared to the other selection cuts, and increases for higher masses (varying between 1.5\% and $7 \%$ 
for masses of the scalar of $20 \mathrm{GeV}$ and $150 \mathrm{GeV}$, respectively). The final cut requires the scalar $S$ candidate invariant mass, $m_{S}^{\text {rec }}$, to be within a mass window of $\pm 30 \mathrm{GeV}$ around the probed value of $m_{S}$.

The cut flow for the signal with an up and with a charm quark is given in tables 1 and 2, respectively. The scalar mass-independent yields for the background components are shown in table 3 while the mass-dependent one is given in table 4. An integrated luminosity of $150 \mathrm{fb}^{-1}$ is considered for this analysis.

Upper limits on the signal cross section are obtained under the signal absence hypothesis, using the $\mathrm{CL}_{\mathrm{s}}$ method [58]. For this, the distribution of the invariant mass of the reconstructed scalar $S$ after all selection cuts is fitted with OpTHyLic [59]. A total of 20 bins per signal point are considered and Poissonian statistical uncertainties on each bin of the distributions are included in the computation. An expected upper limit on the signal strength, $\sigma_{95 \%} / \sigma_{\text {th }}\left(p p \rightarrow t S(q), S \rightarrow \mu^{+} \mu^{-}\right)$, at $95 \%$ confidence level (CL) is then obtained. The signal cross section, $\sigma_{\mathrm{th}}$, is computed with MadGraph v5. The $\pm 1 \sigma$ and $\pm 2 \sigma$ variations are also computed, taking into account the statistical uncertainty arising from finite Monte Carlo samples.

We present the results in figure 4, where we show the 95\% CL upper limits on the top branching ratio $\mathcal{B}\left(t \rightarrow S q, S \rightarrow \mu^{+} \mu^{-}\right)$and cross section $\sigma\left(p p \rightarrow t S(q), S \rightarrow \mu^{+} \mu^{-}\right)$. The $\pm 1 \sigma$ and $\pm 2 \sigma$ bands are plotted in green and yellow, respectively.

Note that the sensitivity worsens at $m_{S} \sim m_{Z}$ due to larger impact of the backgrounds with $Z$-bosons. This effect, however, is attenuated by two factors: (i) the mass window around the singlet mass in which the events are selected is relatively large; and (ii) while the $t Z$ background distribution is larger around $90 \mathrm{GeV}$, the combination of the $t Z$ and $t \bar{t}$ background components is equally important in the neighboring bins of the reconstructed scalar mass distribution.

\section{Search for $t \rightarrow S q, S \rightarrow \tau^{+} \tau^{-}$}

We focus now on the scenario where the scalar $S$ decays to a pair of taus, concentrating on the hadronic decays of the latter. Again, we focus on the leptonic decay of the $W$. Thus, we require events to contain exactly one light lepton and at least three jets, from which exactly one must be $b$-tagged and exactly two must be tagged as taus decaying into hadrons. The efficiency for $\tau$-tagging is $60 \%$ while the misidentification rate is $1 \%$ with no dependency on the transverse momentum. Jets and leptons are defined in the same $p_{T}$ and $|\eta|$ ranges as in the previous analysis; the effect of these requirements in conjunction with the cuts on the number of leptons and hadronic taus can be found in the yields tables, labeled as "basic".

The dominant backgrounds for this channel are the exclusive $t W$ and $t \bar{t}$ processes, where the top quark is assumed to decay to $W b$ and $W \rightarrow \tau \nu$. Indeed, after the aforementioned cuts on the particle multiplicities, the other background components (with the largest cross sections) become irrelevant; see table 7 . 


\begin{tabular}{|l|c|c|c|c|c|c|c|}
\hline Cuts $/ m_{S}$ & $20 \mathrm{GeV}$ & $50 \mathrm{GeV}$ & $80 \mathrm{GeV}$ & $90 \mathrm{GeV}$ & $100 \mathrm{GeV}$ & $120 \mathrm{GeV}$ & $150 \mathrm{GeV}$ \\
\hline basic & $6092 \pm 61$ & $5597 \pm 54$ & $4769 \pm 43$ & $4295 \pm 38$ & $3642 \pm 32$ & $2416 \pm 21$ & $899 \pm 8$ \\
\hline$n_{j}>1$ & $6033 \pm 60$ & $5537 \pm 53$ & $4708 \pm 42$ & $4249 \pm 37$ & $3589 \pm 32$ & $2378 \pm 21$ & $865 \pm 8$ \\
\hline$n_{b}=1$ & $3249 \pm 44$ & $2961 \pm 39$ & $2543 \pm 31$ & $2301 \pm 27$ & $1914 \pm 23$ & $1265 \pm 15$ & $479 \pm 6$ \\
\hline$n_{\mu^{+} \mu^{-}}=1$ & $3247 \pm 44$ & $2959 \pm 39$ & $2542 \pm 31$ & $2300 \pm 27$ & $1913 \pm 23$ & $1265 \pm 15$ & $478 \pm 6$ \\
\hline$\left|m_{t}^{\text {rec }}-m_{t}\right|<50 \mathrm{GeV}$ & $2763 \pm 41$ & $2507 \pm 36$ & $2152 \pm 29$ & $1961 \pm 25$ & $1625 \pm 21$ & $1075 \pm 14$ & $403 \pm 5$ \\
\hline$m_{\text {total }}<1 \mathrm{TeV}$ & $2713 \pm 40$ & $2468 \pm 36$ & $2089 \pm 28$ & $1893 \pm 25$ & $1556 \pm 21$ & $1013 \pm 13$ & $375 \pm 5$ \\
\hline$\left|m_{S}^{\text {rec }}-m_{S}\right|<30 \mathrm{GeV}$ & $1908 \pm 34$ & $1729 \pm 30$ & $1440 \pm 23$ & $1297 \pm 21$ & $1062 \pm 17$ & $690 \pm 11$ & $252 \pm 4$ \\
\hline
\end{tabular}

Table 1. Event yields after each cut for the seven benchmark signal points, in the analysis for $t \rightarrow S u, S \rightarrow \mu^{+} \mu^{-}$. We fix $Y_{13}=Y_{31}=0.1, \Lambda=1 \mathrm{TeV}$ and $\mathcal{B}\left(S \rightarrow \mu^{+} \mu^{-}\right)=1$. The event yields presented assume a collected luminosity of $\mathcal{L}=150 \mathrm{fb}^{-1}$.

\begin{tabular}{|l|c|c|c|c|c|c|c|}
\hline Cuts $/ m_{S}$ & $20 \mathrm{GeV}$ & $50 \mathrm{GeV}$ & $80 \mathrm{GeV}$ & $90 \mathrm{GeV}$ & $100 \mathrm{GeV}$ & $120 \mathrm{GeV}$ & $150 \mathrm{GeV}$ \\
\hline basic & $5235 \pm 51$ & $4923 \pm 46$ & $4070 \pm 35$ & $3633 \pm 31$ & $3050 \pm 26$ & $1836 \pm 15$ & $362 \pm 3$ \\
\hline$n_{j}>1$ & $5214 \pm 51$ & $4906 \pm 46$ & $4050 \pm 35$ & $3616 \pm 31$ & $3030 \pm 26$ & $1821 \pm 15$ & $352 \pm 3$ \\
\hline$n_{b}=1$ & $2705 \pm 37$ & $2520 \pm 33$ & $2103 \pm 25$ & $1870 \pm 22$ & $1571 \pm 18$ & $957 \pm 11$ & $188 \pm 2$ \\
\hline$n_{\mu^{+} \mu^{-}}=1$ & $2705 \pm 37$ & $2520 \pm 33$ & $2102 \pm 25$ & $1870 \pm 22$ & $1571 \pm 18$ & $957 \pm 11$ & $188 \pm 2$ \\
\hline$\left|m_{t}^{\text {rec }}-m_{t}\right|<50 \mathrm{GeV}$ & $2229 \pm 33$ & $2072 \pm 30$ & $1754 \pm 23$ & $1551 \pm 20$ & $1311 \pm 17$ & $801 \pm 10$ & $161 \pm 2$ \\
\hline$m_{\text {total }}<1 \mathrm{TeV}$ & $2194 \pm 33$ & $2038 \pm 29$ & $1708 \pm 23$ & $1488 \pm 20$ & $1248 \pm 16$ & $749 \pm 10$ & $148 \pm 2$ \\
\hline$\left|m_{S}^{\text {rec }}-m_{S}\right|<30 \mathrm{GeV}$ & $1502 \pm 28$ & $1406 \pm 24$ & $1166 \pm 19$ & $1003 \pm 16$ & $829 \pm 13$ & $497 \pm 8$ & $100 \pm 2$ \\
\hline
\end{tabular}

Table 2. Event yields after each cut for the seven benchmark signal points, in the analysis for $t \rightarrow S c, S \rightarrow \mu^{+} \mu^{-}$. We fix $Y_{23}=Y_{32}=0.1, \Lambda=1 \mathrm{TeV}$ and $\mathcal{B}\left(S \rightarrow \mu^{+} \mu^{-}\right)=1$. The event yields presented assume a collected luminosity of $\mathcal{L}=150 \mathrm{fb}^{-1}$.

\begin{tabular}{|l|c|c|c|c|c|c|}
\hline Cuts/Background & $t W$ & $t \bar{t} W / t \bar{t} Z$ & $Z Z Z / W W Z$ & $Z Z / W Z / W W$ & $t \bar{t}$ & $t Z$ \\
\hline basic & $334 \pm 236$ & $5.2 \pm 0.7$ & $10.1 \pm 0.5$ & $16615 \pm 2220$ & $348 \pm 24$ & $128 \pm 2$ \\
\hline$n_{j}>1$ & $334 \pm 236$ & $5.2 \pm 0.7$ & $9.1 \pm 0.5$ & $8011 \pm 1542$ & $326 \pm 24$ & $128 \pm 2$ \\
\hline$n_{b}=1$ & $334 \pm 236$ & $2.2 \pm 0.5$ & $1.0 \pm 0.2$ & $<74$ & $172 \pm 17$ & $65 \pm 1$ \\
\hline$n_{\mu^{+} \mu^{-}}=1$ & $<42$ & $1.1 \pm 0.3$ & $0.7 \pm 0.1$ & - & $172 \pm 17$ & $39 \pm 1$ \\
\hline$\left|m_{t}^{\text {rec }}-m_{t}\right|<50 \mathrm{GeV}$ & - & $0.7 \pm 0.3$ & $0.4 \pm 0.1$ & - & $114 \pm 14$ & $31 \pm 1$ \\
\hline$m_{\text {total }}<1 \mathrm{TeV}$ & - & $0.3 \pm 0.2$ & $0.25 \pm 0.08$ & - & $80 \pm 12$ & $23.2 \pm 0.9$ \\
\hline
\end{tabular}

Table 3. Event yields after each cut for the dominant backgrounds, in the analysis for $t \rightarrow S q, S \rightarrow$ $\mu^{+} \mu^{-}$. The $Z+$ jets sample is reduced to negligible values after the cut on the lepton multiplicity. The event yields presented assume a collected luminosity of $\mathcal{L}=150 \mathrm{fb}^{-1}$.

\begin{tabular}{|l|c|c|c|c|c|c|c|}
\hline Background $/ m_{S}$ & $20 \mathrm{GeV}$ & $50 \mathrm{GeV}$ & $80 \mathrm{GeV}$ & $90 \mathrm{GeV}$ & $100 \mathrm{GeV}$ & $120 \mathrm{GeV}$ & $150 \mathrm{GeV}$ \\
\hline$t \bar{t} W / t \bar{t} Z$ & $<0.09$ & $<0.09$ & $0.2 \pm 0.1$ & $0.2 \pm 0.1$ & $0.2 \pm 0.1$ & $0.2 \pm 0.1$ & $<0.09$ \\
\hline$Z Z Z / W W Z$ & $0 \pm 0$ & $0.06 \pm 0.04$ & $0.22 \pm 0.08$ & $0.17 \pm 0.07$ & $0.17 \pm 0.07$ & $<0.03$ & $<0.03$ \\
\hline$t \bar{t}(\mu)$ & $12 \pm 4$ & $34 \pm 8$ & $39 \pm 8$ & $39 \pm 8$ & $36 \pm 8$ & $15 \pm 5$ & $3 \pm 2$ \\
\hline$t Z$ & $1.8 \pm 0.2$ & $3.3 \pm 0.3$ & $18.6 \pm 0.8$ & $18.4 \pm 0.8$ & $17.9 \pm 0.8$ & $11.4 \pm 0.6$ & $0.8 \pm 0.2$ \\
\hline
\end{tabular}

Table 4. Event yields for the last selection cut on $m_{S}^{\text {rec }}$ for the dominant backgrounds, in the analysis for $t \rightarrow S q, S \rightarrow \mu^{+} \mu^{-}$. The event yields presented assume a collected luminosity of $\mathcal{L}=150 \mathrm{fb}^{-1}$. 

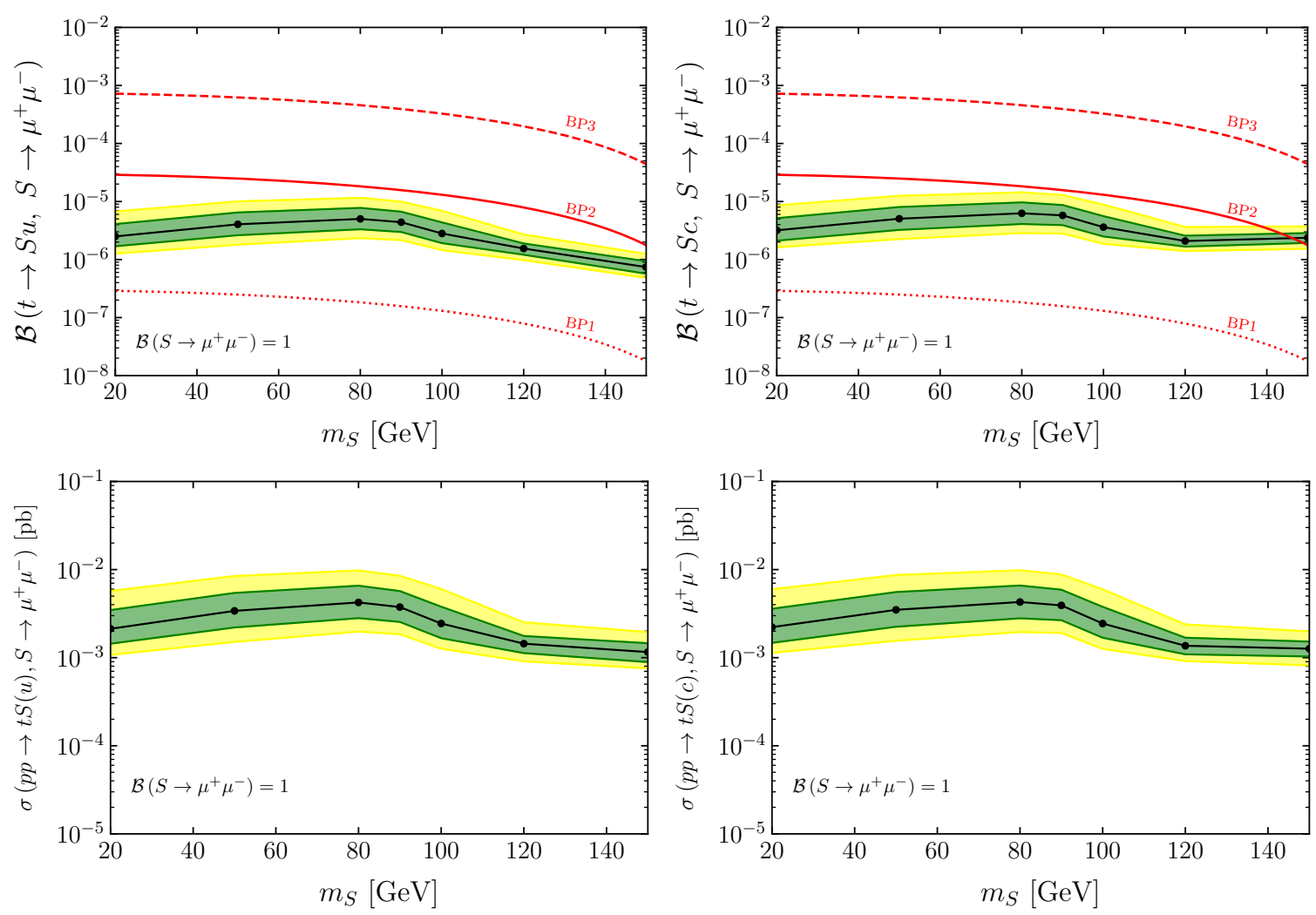

Figure 4. In the upper (bottom) panels, we show the 95\% CL limits on the branching ratio (cross section times branching ratio) that can be tested in the $\mu^{+} \mu^{-}$channel, in the analysis proposed for $t \rightarrow S q, S \rightarrow \mu^{+} \mu^{-}$, with $q=u(c)$ in the panels on the left (right). The limits are obtained for a collected luminosity $\mathcal{L}=150 \mathrm{fb}^{-1}$. The green and yellow bands show the $\pm 1 \sigma$ and $\pm 2 \sigma$ uncertainty on the limits, respectively. Superimposed are the theoretical expectations in three BPs.

We (partially) reconstruct the scalar $S$ from the two tau-jets obtaining its invariant mass, $m_{S}^{\text {rec }}$. In the left panels of figure 5 we show the normalized distribution of this variable, after the basic selection cuts, in two signal BPs and in the main backgrounds. In the same figure, we plot the transverse mass distribution of the system composed by the lepton, the $b$-tagged jet, the reconstructed scalar and the missing energy. We require this latter variable to be smaller than $500 \mathrm{GeV}$. Finally, events are required to fulfill $\left|m_{S}-m_{S}^{\mathrm{rec}}\right|<30 \mathrm{GeV}$, where $m_{S}^{\mathrm{rec}}$ is the mass of the reconstructed scalar candidate and $m_{S}$ is the corresponding value being probed.

The cut flows for the signal in the up quark and in the charm quark cases are given in tables 5 and 6 ; respectively. Likewise, table 7 shows the $m_{S}$-independent yields for the different backgrounds and in table 8 we write the mass-dependent ones. Similarly to the previous analysis, the expected upper limit on the signal strength, $\sigma_{95 \%} / \sigma_{\text {th }}(p p \rightarrow t S(q)$, $S \rightarrow \tau^{+} \tau^{-}$), is obtained using the invariant mass of the scalar $S$ candidate distributed into 20 bins. The $95 \%$ CL upper limits on the branching ratio $\mathcal{B}\left(t \rightarrow S q, S \rightarrow \tau^{+} \tau^{-}\right)$and on the cross section $\sigma\left(p p \rightarrow t S(q), S \rightarrow \tau^{+} \tau^{-}\right)$are shown in figure 6 . 


\begin{tabular}{|l|c|c|c|c|c|c|c|}
\hline Cuts $/ m_{S}$ & $20 \mathrm{GeV}$ & $50 \mathrm{GeV}$ & $80 \mathrm{GeV}$ & $90 \mathrm{GeV}$ & $100 \mathrm{GeV}$ & $120 \mathrm{GeV}$ & $150 \mathrm{GeV}$ \\
\hline basic & $267 \pm 13$ & $596 \pm 17$ & $586 \pm 15$ & $561 \pm 14$ & $469 \pm 11$ & $339 \pm 8$ & $131 \pm 3$ \\
\hline$n_{j}>3$ & $264 \pm 13$ & $588 \pm 17$ & $578 \pm 15$ & $553 \pm 13$ & $461 \pm 11$ & $331 \pm 8$ & $126 \pm 3$ \\
\hline$n_{b}=1$ & $118 \pm 8$ & $306 \pm 12$ & $309 \pm 11$ & $330 \pm 10$ & $284 \pm 8$ & $171 \pm 6$ & $66 \pm 2$ \\
\hline$m_{T}<500 \mathrm{GeV}$ & $86 \pm 7$ & $259 \pm 11$ & $256 \pm 910$ & $280 \pm 10$ & $234 \pm 8$ & $143 \pm 5$ & $47 \pm 2$ \\
\hline$\left|m_{S}^{\text {rec }}-m_{S}\right|<30 \mathrm{GeV}$ & $47 \pm 5$ & $220 \pm 11$ & $193 \pm 9$ & $236 \pm 9$ & $195 \pm 7$ & $79 \pm 4$ & $18 \pm 1$ \\
\hline
\end{tabular}

Table 5. Event yields after each cut for the seven benchmark signal points, in the analysis for $t \rightarrow S u, S \rightarrow \tau^{+} \tau^{-}$. We use $Y_{13}=Y_{31}=0.1, \Lambda=1 \mathrm{TeV}$ and $\mathcal{B}\left(S \rightarrow \tau^{+} \tau^{-}\right)=1$. The event yields presented assume a collected luminosity of $\mathcal{L}=150 \mathrm{fb}^{-1}$.

\begin{tabular}{|l|c|c|c|c|c|c|c|}
\hline Cuts $/ m_{S}$ & $20 \mathrm{GeV}$ & $50 \mathrm{GeV}$ & $80 \mathrm{GeV}$ & $90 \mathrm{GeV}$ & $100 \mathrm{GeV}$ & $120 \mathrm{GeV}$ & $150 \mathrm{GeV}$ \\
\hline basic & $221 \pm 10$ & $540 \pm 15$ & $486 \pm 12$ & $462 \pm 11$ & $410 \pm 9$ & $263 \pm 6$ & $54 \pm 1$ \\
\hline$n_{j}>3$ & $219 \pm 10$ & $536 \pm 15$ & $482 \pm 12$ & $458 \pm 11$ & $407 \pm 9$ & $260 \pm 6$ & $52 \pm 1$ \\
\hline$n_{b}=1$ & $107 \pm 7$ & $276 \pm 11$ & $256 \pm 9$ & $297 \pm 8$ & $215 \pm 7$ & $131 \pm 4$ & $27.5 \pm 0.8$ \\
\hline$m_{T}<500 \mathrm{GeV}$ & $91 \pm 7$ & $243 \pm 10$ & $222 \pm 8$ & $244 \pm 8$ & $182 \pm 6$ & $111 \pm 4$ & $22.9 \pm 0.7$ \\
\hline$\left|m_{S}^{\text {rec }}-m_{S}\right|<30 \mathrm{GeV}$ & $48 \pm 5$ & $203 \pm 9$ & $171 \pm 7$ & $207 \pm 7$ & $124 \pm 5$ & $62 \pm 3$ & $9.0 \pm 0.5$ \\
\hline
\end{tabular}

Table 6. Event yields after each cut for the seven benchmark signal points, in the analysis for $t \rightarrow S c, S \rightarrow \tau^{+} \tau^{-}$. We fix $Y_{23}=Y_{32}=0.1, \Lambda=1 \mathrm{TeV}$ and $\mathcal{B}\left(S \rightarrow \tau^{+} \tau^{-}\right)=1$. The event yields presented assume a collected luminosity of $\mathcal{L}=150 \mathrm{fb}^{-1}$.

\begin{tabular}{|l|c|c|c|c|c|c|}
\hline Cuts/Background & $t W(\tau)$ & $t \bar{t} W / t \bar{t} Z$ & $Z Z Z / W W Z$ & $Z Z / W Z / W W$ & $t \bar{t}(\tau)$ & $t Z$ \\
\hline basic & $48 \pm 4$ & $1.0 \pm 0.3$ & $1.4 \pm 0.2$ & $297 \pm 133$ & $99 \pm 5$ & $1.3 \pm 0.2$ \\
\hline$n_{j}>3$ & $44 \pm 3$ & $1.0 \pm 0.3$ & $1.2 \pm 0.2$ & $178 \pm 103$ & $96 \pm 5$ & $1.3 \pm 0.2$ \\
\hline$n_{b}=1$ & $19 \pm 2$ & $0.6 \pm 0.2$ & $0.06 \pm 0.04$ & $<74$ & $52 \pm 4$ & $0.4 \pm 0.1$ \\
\hline$m_{T}<500 \mathrm{GeV}$ & $11 \pm 2$ & $0.4 \pm 0.2$ & $0.05 \pm 0.04$ & - & $42 \pm 3$ & $0.3 \pm 0.1$ \\
\hline
\end{tabular}

Table 7. Event yields after each cut for the dominant backgrounds, in the analysis for $t \rightarrow S q, S \rightarrow$ $\tau^{+} \tau^{-}$. The $Z+$ jets sample is reduced to negligible values after the fifth cut. The event yields presented assume a collected luminosity of $\mathcal{L}=150 \mathrm{fb}^{-1}$.

\begin{tabular}{|l|c|c|c|c|c|c|c|}
\hline Background $/ m_{S}$ & $20 \mathrm{GeV}$ & $50 \mathrm{GeV}$ & $80 \mathrm{GeV}$ & $90 \mathrm{GeV}$ & $100 \mathrm{GeV}$ & $120 \mathrm{GeV}$ & $150 \mathrm{GeV}$ \\
\hline$t W(\tau)$ & $0.6 \pm 0.4$ & $3.1 \pm 0.9$ & $5 \pm 1$ & $5 \pm 1$ & $5 \pm 1$ & $5 \pm 1$ & $4 \pm 1$ \\
\hline$t \bar{t} W / t \bar{t} Z$ & $<0.09$ & $<0.09$ & $0.3 \pm 0.2$ & $0.3 \pm 0.2$ & $0.2 \pm 0.1$ & $<0.09$ & $<0.09$ \\
\hline$Z Z Z / W W Z$ & $<0.03$ & $<0.03$ & $<0.03$ & $<0.03$ & $<0.03$ & $<0.03$ & $<0.03$ \\
\hline$t \bar{t}(\tau)$ & $3.7 \pm 0.9$ & $17 \pm 2$ & $24 \pm 2$ & $21 \pm 2$ & $20 \pm 2$ & $14 \pm 2$ & $10 \pm 1$ \\
\hline$t Z$ & $<0.03$ & $0.07 \pm 0.05$ & $0.10 \pm 0.06$ & $0.10 \pm 0.06$ & $0.10 \pm 0.06$ & $0.10 \pm 0.06$ & $0.14 \pm 0.07$ \\
\hline
\end{tabular}

Table 8. Event yields for the last selection cut on $m_{S}^{\text {rec }}$ for the dominant backgrounds, in the analysis for $t \rightarrow S q, S \rightarrow \tau^{+} \tau^{-}$. The event yields presented assume a collected luminosity of $\mathcal{L}=150 \mathrm{fb}^{-1}$. 

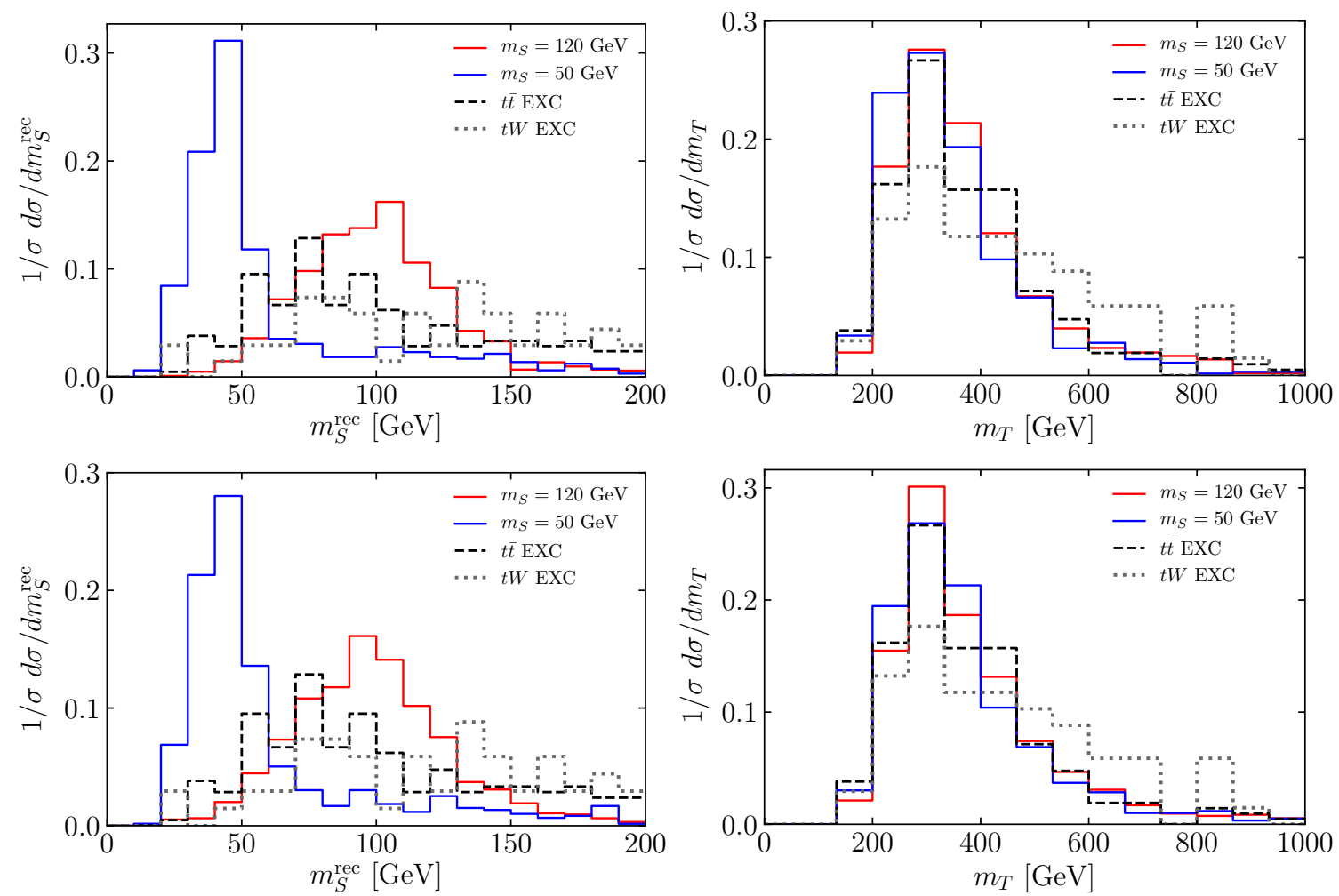

Figure 5. The reconstructed scalar mass (left) and the transverse mass of the system composed by the lepton, the $b$-jet, the reconstructed $S$ and missing energy (right) in the analysis proposed for $t \rightarrow S q, S \rightarrow \tau^{+} \tau^{-}$. In the upper (bottom) panel, $q=c(u)$. We represent the distributions of two signal benchmark points and the two major background components, after the cut on the particle multiplicities; the background samples are generated exclusively, i.e. only gauge boson decays into taus are included. The distributions assume a collected luminosity of $\mathcal{L}=150 \mathrm{fb}^{-1}$.

\section{Search for $t \rightarrow S S q, S \rightarrow \mu^{+} \mu^{-}$}

Finally, let us develop an analysis to search for two singlet scalars produced in association with a top quark (or in the decay of a top quark in pair production), both decaying to a pair of muons. We focus on the hadronic decays of the $W$. The final state consists of four isolated leptons and at least three jets, one of them required to be $b$-tagged. Jets and leptons are defined in the same $p_{T}$ and $|\eta|$ ranges as before; the effect of these requirements, together with the cut on the lepton multiplicity, can be found in the yields tables labeled as "basic". Due to the large lepton multiplicity, all the background components are significantly reduced; with the additional cuts on the number of jets, most become negligible. We are left with six background events from the $t \bar{t} V$ and $t \bar{t}$ exclusive samples; see table 11.

We reconstruct the top quark from a $W$ boson and a $b$-jet; its invariant mass being $m_{t}^{\text {rec }}$. The $W$ is reconstructed from the two light jets with invariant mass closest to $m_{W}$. We then require $m_{t}^{\text {rec }}$ to be within a window of $50 \mathrm{GeV}$ around the top mass. The two scalars $S$ candidates are reconstructed by requiring two muons with opposite sign, with the event being rejected if no such candidates are found. The opposite-sign muons reconstructing the 

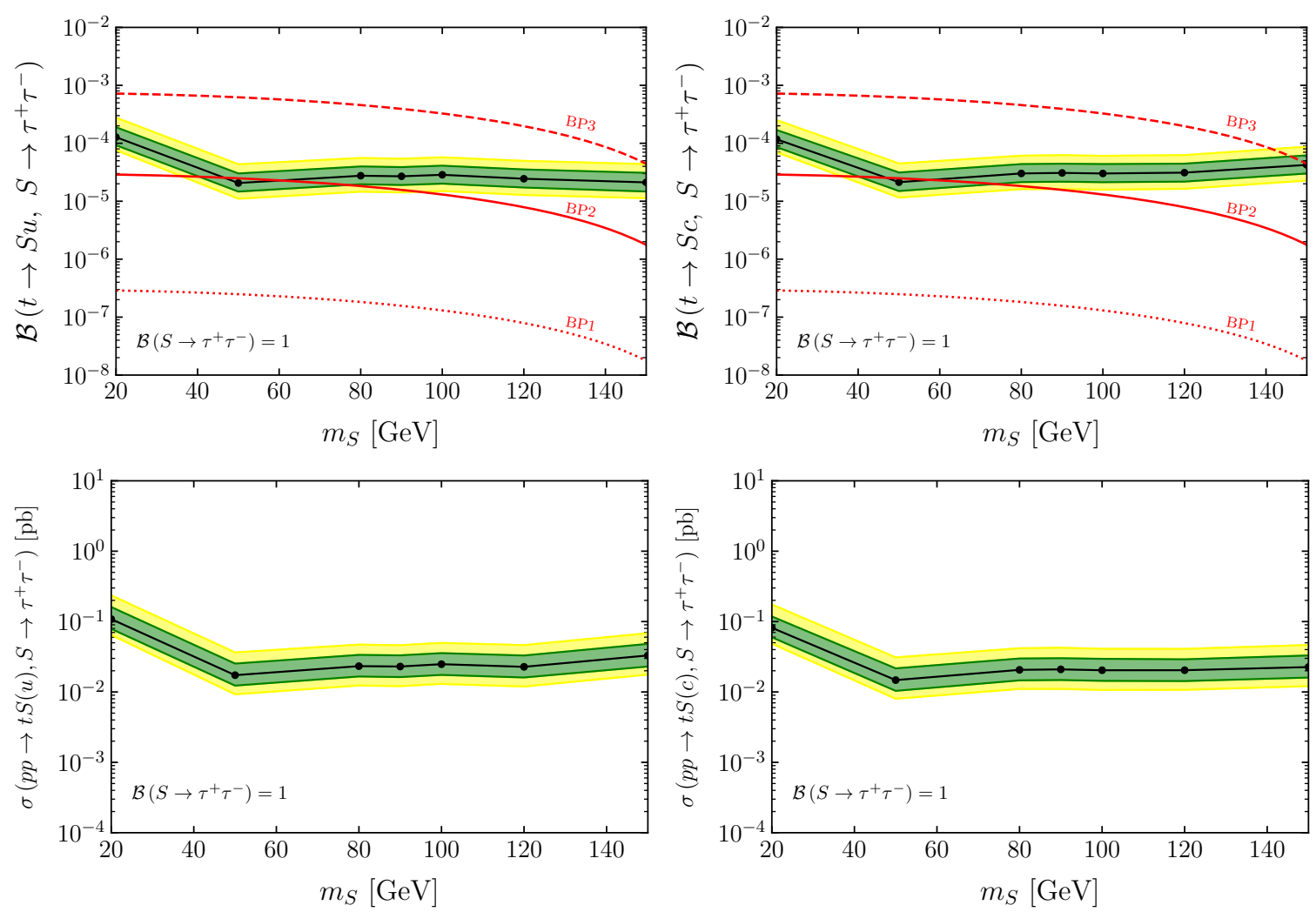

Figure 6. In the upper (bottom) panels, we show the 95\% CL limits on the branching ratio (cross section times branching ratio) that can be tested in the $\tau^{+} \tau^{-}$channel, in the analysis proposed for $t \rightarrow S q, S \rightarrow \tau^{+} \tau^{-}$, with $q=u(c)$ in the panels on the left (right). The green and yellow bands show the $\pm 1 \sigma$ and $\pm 2 \sigma$ uncertainty on the limits, respectively. The limits are obtained for a collected luminosity $\mathcal{L}=150 \mathrm{fb}^{-1}$. Superimposed are the theoretical expectations in three BPs.

two scalars are those minimizing $\left|m_{S_{1}}^{\mathrm{rec}}-m_{S_{2}}^{\mathrm{rec}}\right|$, with $m_{S_{1,2}}^{\mathrm{rec}}$ being the invariant mass of each pair of opposite sign muons. It is also required that the invariant mass of the total system, composed of the reconstructed top quark and the two scalars, is smaller than $1 \mathrm{TeV}$. We finally request the $m_{S_{1,2}}^{\text {rec }}$ to be within a window of $30 \mathrm{GeV}$ from the mass of $S$ being probed.

In figures 7 and 8, we show the normalized distributions of $m_{t}^{\mathrm{rec}}$ and each $m_{S}^{\mathrm{rec}}$, respectively, for two signal benchmark points after the basic selection cuts.

The cut flows for the signal are given in tables 9 and 10; in table 11 we show the scalar mass-independent cut flow for the relevant backgrounds. With the final cut on $m_{S_{1,2}}^{\text {rec }}$, the analysis becomes essentially background-free. As before, expected upper limits on the signal strength, $\sigma_{95 \%} / \sigma_{\text {th }}\left(p p \rightarrow t S S(q), S \rightarrow \mu^{+} \mu^{-}\right)$, are obtained using the invariant mass of the scalar $S$ candidate distributed into 20 bins. The $95 \%$ upper limits on the branching ratio $\mathcal{B}\left(t \rightarrow S S q, S \rightarrow \mu^{+} \mu^{-}\right)$and cross section $\sigma\left(p p \rightarrow t S S(q), S \rightarrow \mu^{+} \mu^{-}\right)$are shown in figure 9 , again including the $\pm 1 \sigma$ (green band) and the $\pm 2 \sigma$ (yellow band) uncertainties. 

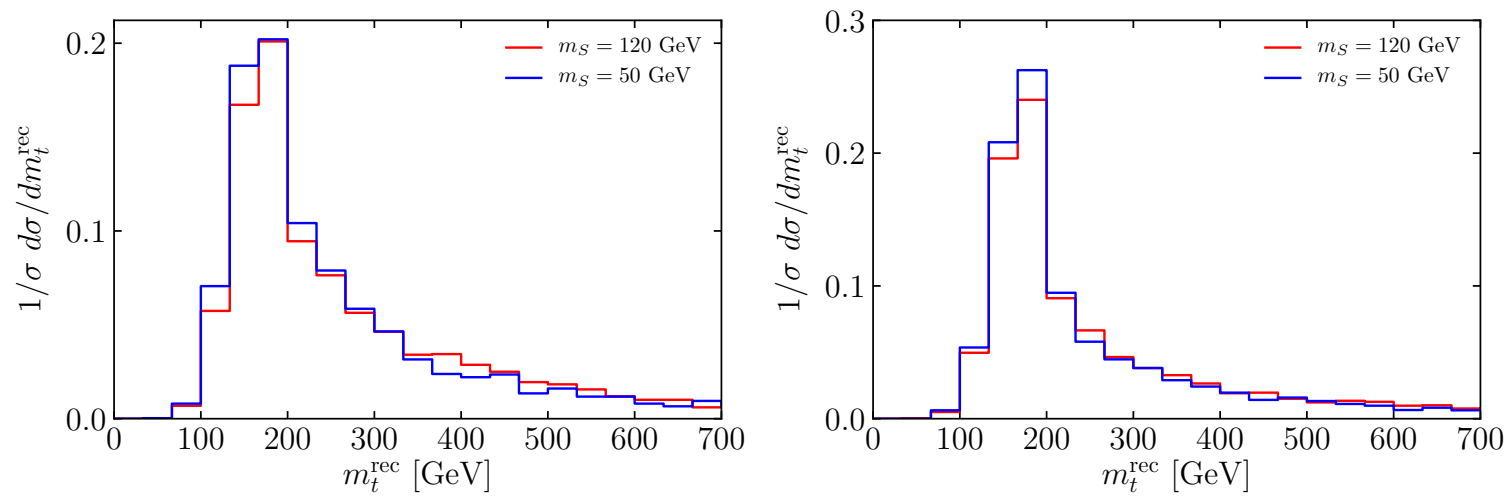

Figure 7. The reconstructed top mass in the analysis proposed for $t \rightarrow S S q, S \rightarrow \mu^{+} \mu^{-}$. In the left (right) panel, $q=c(u)$. The distributions assume a collected luminosity of $\mathcal{L}=150 \mathrm{fb}^{-1}$.
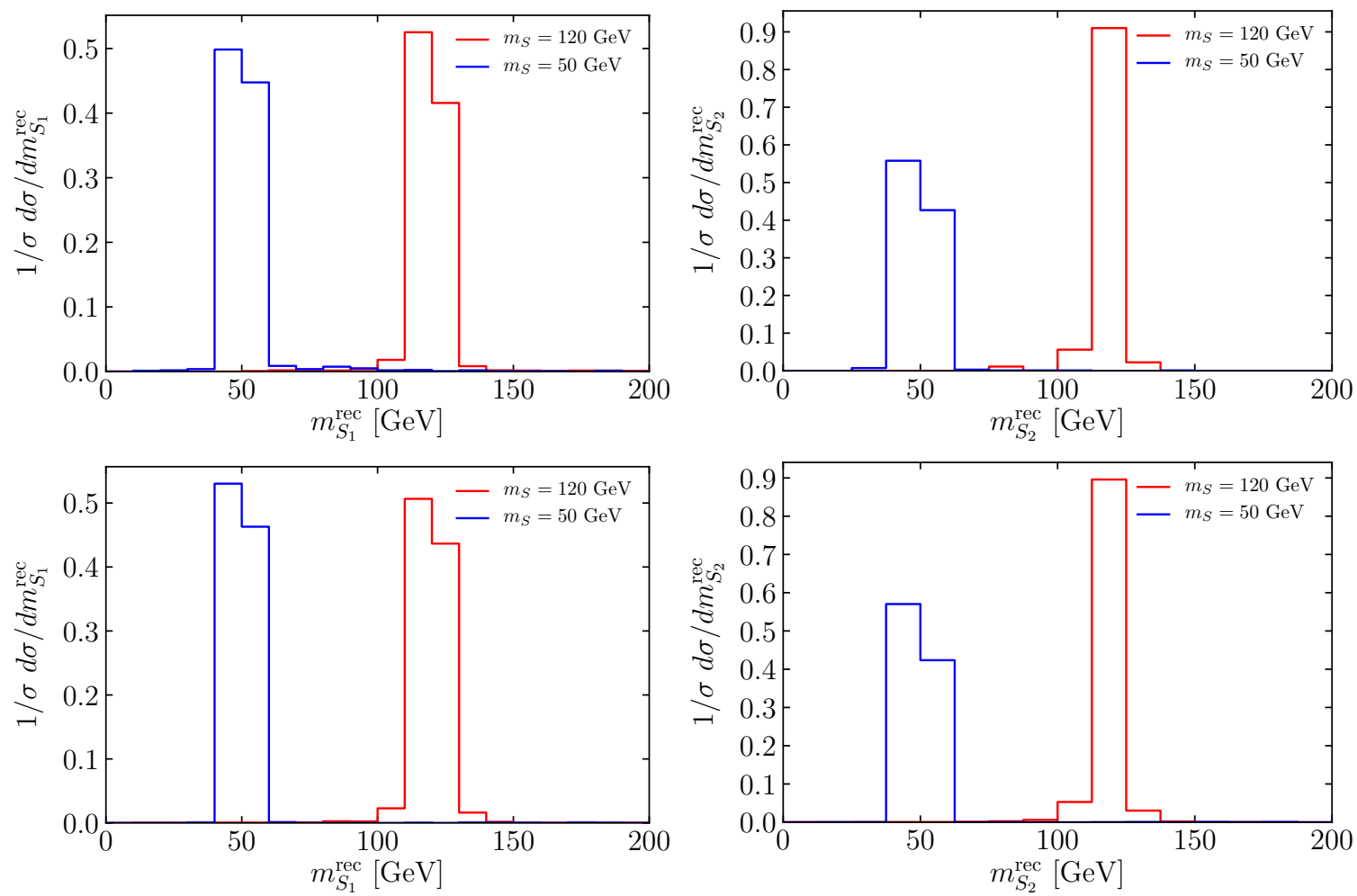

Figure 8. The two reconstructed scalar masses, $m_{S_{1}}^{\text {rec }}$ and $m_{S_{2}}^{\text {rec }}$, in the analysis proposed for $t \rightarrow S S q, S \rightarrow \mu^{+} \mu^{-}$. In the upper (bottom) panel, $q=c(u)$. The distributions assume a collected luminosity of $\mathcal{L}=150 \mathrm{fb}^{-1}$. 


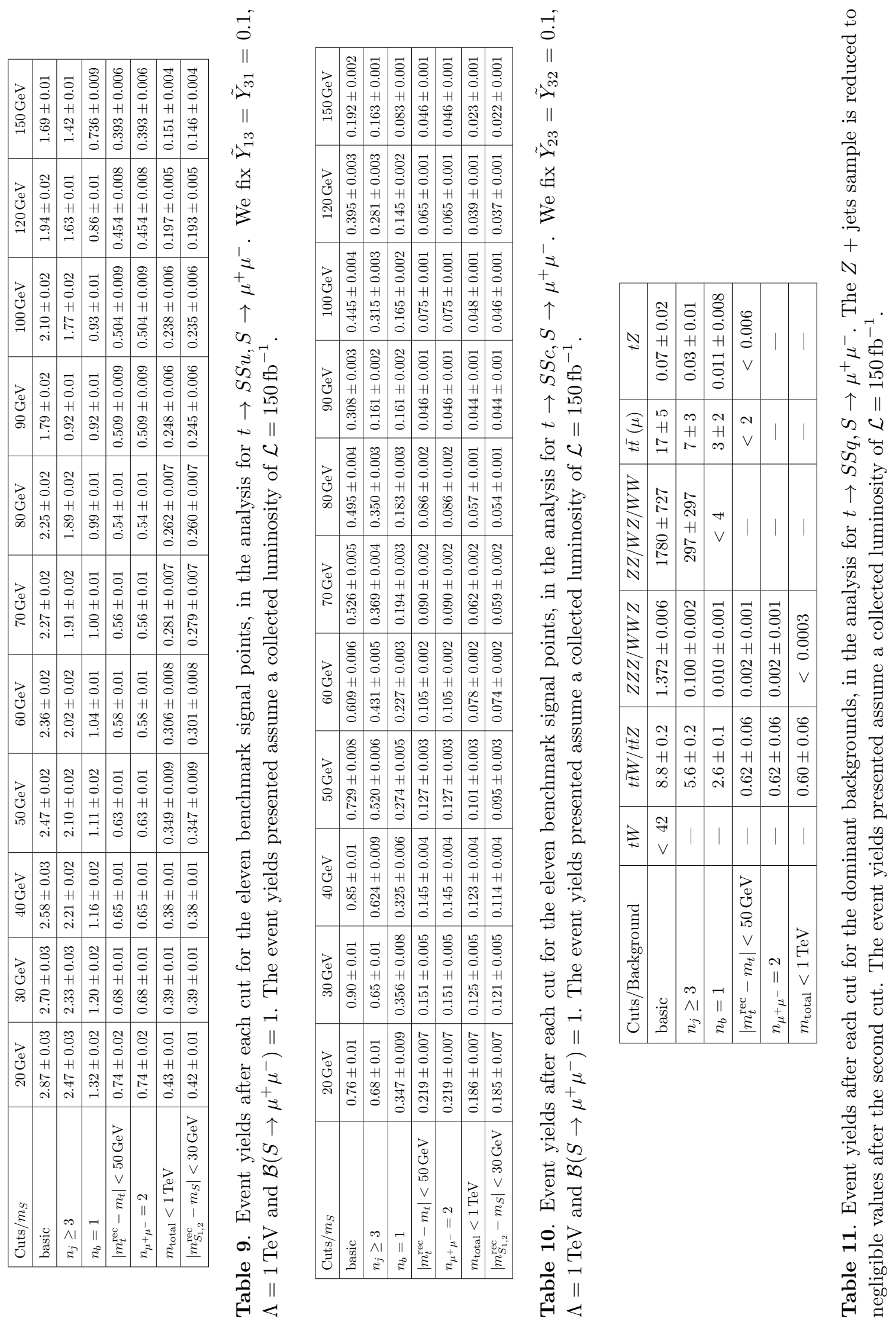



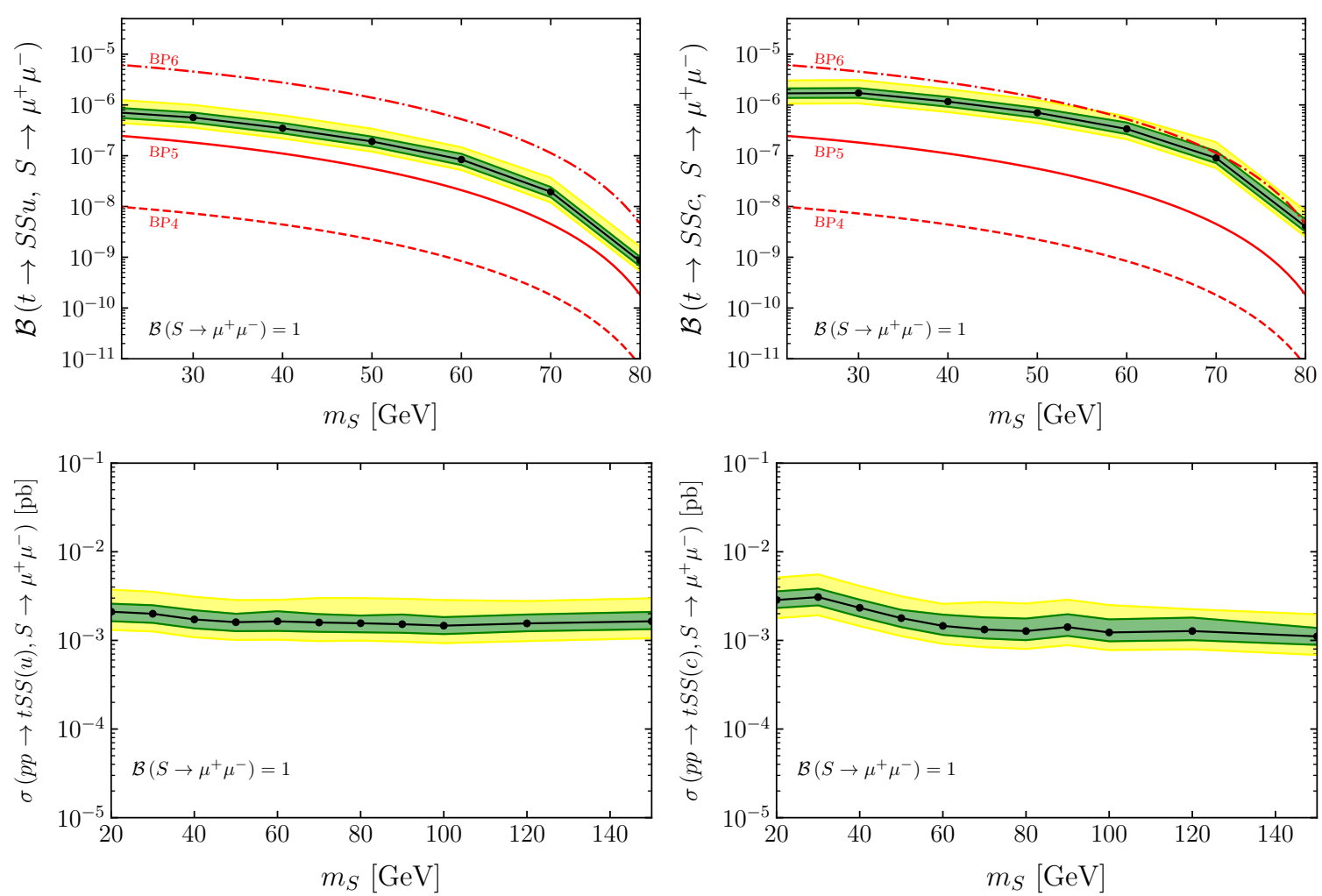

Figure 9. In the upper (bottom) panels, we show the 95\% CL limits on the branching ratio (cross section times branching ratio) that can be tested in the $\mu^{+} \mu^{-}$channel, in the analysis proposed for $t \rightarrow S S q, S \rightarrow \mu^{+} \mu^{-}$, with $q=u(c)$ in the panels on the left (right). The green and yellow bands show the $\pm 1 \sigma$ and $\pm 2 \sigma$ uncertainty on the limits, respectively. The limits are obtained for a collected luminosity $\mathcal{L}=150 \mathrm{fb}^{-1}$. Superimposed are the theoretical expectations in three BPs.

\section{Conclusions}

Using an effective field theory approach, we have shown that, in models of new physics with light pseudo-scalar singlet degrees of freedom $S$, new top flavor-changing neutral currents (FCNCs) arise. These can trigger the rare top decay $t \rightarrow S q$, as well as produce the top quark in association with $S$ in proton-proton collisions. At the LHC, both processes can be captured by inclusive searches for $p p \rightarrow t S+j$.

We have shown that, if there is a $\mathbb{Z}_{2}$ symmetry under which $S$ is odd while all the SM quarks are even, then one expects rather the FCNC process $p p \rightarrow t S S+j$. Under this hypothesis, $S$ decays exclusively into leptons, predominantly into taus and muons. For completeness we have shown how these ideas can be implemented in a concrete composite Higgs model. We have also demonstrated that experimental analyses currently performed at the LHC are not significantly sensitive to these interactions. Thus, we have worked out three new dedicated searches in full detail, including detector simulation and with a rigorous quantification of uncertainties, to probe the processes $p p \rightarrow t S, S \rightarrow \mu^{+} \mu^{-}$; $p p \rightarrow t S, S \rightarrow \tau^{+} \tau^{-}$and $p p \rightarrow t S S, S \rightarrow \mu^{+} \mu^{-}$. 
In the channel $p p \rightarrow t S, S \rightarrow \mu^{+} \mu^{-}$, we focus on final state events with three light leptons and jets (with exactly one $b$-tagged). The main discriminating variable is the invariant mass of the hardest opposite sign muon pair. The dominant background ensues from $t \bar{t}$ and $t Z$. For an integrated luminosity of $150 \mathrm{fb}^{-1}$, we find that a production cross section $\sigma\left(p p \rightarrow t S, S \rightarrow \mu^{+} \mu^{-}\right)>10^{-3} \mathrm{pb}$ can be tested at the $95 \% \mathrm{CL}$. The highest sensitivity is attained for $m_{S} \sim 150 \mathrm{GeV}$, for which the maximum number of events compatible with background fluctuations is found to be $\sim 170$ (190) in the up (charm) channel. Considering a new physics coupling and scale $\Lambda$ equal to 0.1 and $5 \mathrm{TeV}$, we predict $\sim 410$ (140) signal events, assuming that $\mathcal{B}\left(S \rightarrow \mu^{+} \mu^{-}\right)=1$. Therefore, this benchmark point could be excluded in the analysis with the up quark. For the same singlet mass, we can probe $\mathcal{B}(t \rightarrow S q)>5(15) \times 10^{-7}$ at the $95 \% \mathrm{CL}$. In turn, for $\mathcal{O}(1)$ couplings in the $\mathrm{UV}$, these results translate into a lower bound on $\Lambda \sim 90 \mathrm{TeV}$.

In the channel $p p \rightarrow t S, S \rightarrow \tau^{+} \tau^{-}$, we focus on final state events with one light lepton and jets (with one $b$-tagged and two hadronic taus). The main discriminating variable is the invariant mass of the two tau-jets. The dominant backgrounds are $t \bar{t}$ and $t W$. For an integrated luminosity of $150 \mathrm{fb}^{-1}$, we find that a production cross section $\sigma\left(p p \rightarrow t S, S \rightarrow \tau^{+} \tau^{-}\right)>10^{-2} \mathrm{pb}$ can be tested at the $95 \%$ CL. The highest sensitivity is attained for $m_{S} \sim 50 \mathrm{GeV}$, for which the maximum number of events is found to be $\sim 2.6(2.2) \times 10^{3}$ in the up (charm) channel. Considering a new physics coupling and scale equal to 0.1 and $1 \mathrm{TeV}$, we predict $\sim 7.8(6.5) \times 10^{4}$ signal events, assuming that $\mathcal{B}\left(S \rightarrow \tau^{+} \tau^{-}\right)=1$. Therefore, this benchmark point could be excluded. For the same singlet mass, we can probe $\mathcal{B}(t \rightarrow S q)>11(12) \times 10^{-6}$ at the $95 \%$ CL. In turn, for $\mathcal{O}(1)$ couplings in the UV, these results translate into a lower bound on $\Lambda \sim 75 \mathrm{TeV}$.

For $p p \rightarrow t S S, S \rightarrow \mu^{+} \mu^{-}$, we concentrate on events with four light leptons and jets (one of which $b$-tagged). The principal discriminating variable is the invariant mass of the four leptons. This search is in good approximation background free. For an integrated luminosity of $150 \mathrm{fb}^{-1}$, we find that a production cross section $\sigma\left(p p \rightarrow t S S, S \rightarrow \mu^{+} \mu^{-}\right)>$ $10^{-3} \mathrm{pb}$ can be tested at $95 \% \mathrm{CL}$. For $m_{S} \sim 80 \mathrm{GeV}$, the maximum number of events is found to be $\sim 230$ (190) in the up (charm) channel. Considering a new physics coupling and scale equal to 1.0 and $1 \mathrm{TeV}$, we predict $\sim 1280(220)$ signal events, assuming that $\mathcal{B}\left(S \rightarrow \mu^{+} \mu^{-}\right)=1$. Therefore, this benchmark point could be excluded. The strongest limits in branching ratio, $\mathcal{B}(t \rightarrow S S q)>5(25) \times 10^{-10}$, are obtained for this mass point, while we can probe $\mathcal{B}(t \rightarrow S S q)>10^{-6}$ at the $95 \%$ CL in the small mass regime. In turn, for $\mathcal{O}(1)$ couplings in the UV, these results translate into a lower bound on $\Lambda \sim 2 \mathrm{TeV}$.

Naive prospects for higher luminosities can be obtained by scaling the statistical significance with $\sqrt{\mathcal{L}}$. Thus, at $\mathcal{L}=3 \mathrm{ab}^{-1}$, we expect to probe scales of order 200, 160, and $3 \mathrm{TeV}$ in each of the channels, respectively. 


\section{Acknowledgments}

The authors were partially funded by FCT - Fundação para a Ciência e a Tecnologia, I.P., Portugal, under projects CERN/FIS-PAR/0024/2019 (MC, NC and MR) and CERN/FIS$\mathrm{PAR} / 0002 / 2019$ (NC and AP). MC is also supported by the Spanish MINECO under the Juan de la Cierva programme as well as by the Ministry of Science and Innovation under grant numbers FPA2016-78220-C3-3-P (fondos FEDER), and by the Junta de Andalucía grants FQM 101 and A-FQM-211-UGR-18 (fondos FEDER). AP acknowledges the support by FCT through grant SFRH/BD/129321/2017. MR acknowledges support by FCT under the grant PD/BD/142773/2018 and by LIP (FCT, COMPETE2020-Portugal2020, FEDER, POCI-01-0145-FEDER-007334). The computational part of this work was supported by INCD (funded by FCT and FEDER under the project 01/SAICT/2016 nr. 022153) and by the Minho Advanced Computing Center (MACC).

Open Access. This article is distributed under the terms of the Creative Commons Attribution License (CC-BY 4.0), which permits any use, distribution and reproduction in any medium, provided the original author(s) and source are credited.

\section{References}

[1] U. Ellwanger, C. Hugonie and A.M. Teixeira, The Next-to-Minimal Supersymmetric Standard Model, Phys. Rept. 496 (2010) 1 [arXiv:0910.1785] [InSPIRE].

[2] R. Contino, Y. Nomura and A. Pomarol, Higgs as a holographic pseudoGoldstone boson, Nucl. Phys. B 671 (2003) 148 [hep-ph/0306259] [INSPIRE].

[3] B. Gripaios, A. Pomarol, F. Riva and J. Serra, Beyond the Minimal Composite Higgs Model, JHEP 04 (2009) 070 [arXiv:0902.1483] [INSPIRE].

[4] M. Chala, $h \rightarrow \gamma \gamma$ excess and Dark Matter from Composite Higgs Models, JHEP 01 (2013) 122 [arXiv: 1210.6208] [INSPIRE].

[5] L. Vecchi, The Natural Composite Higgs, arXiv:1304.4579 [INSPIRE].

[6] B. Bellazzini, C. Csáki and J. Serra, Composite Higgses, Eur. Phys. J. C 74 (2014) 2766 [arXiv: 1401.2457] [INSPIRE].

[7] V. Sanz and J. Setford, Composite Higgses with seesaw EWSB, JHEP 12 (2015) 154 [arXiv: 1508.06133] [INSPIRE].

[8] T. Ma and G. Cacciapaglia, Fundamental Composite 2HDM: SU(N) with 4 flavours, JHEP 03 (2016) 211 [arXiv: 1508.07014] [INSPIRE].

[9] A. Belyaev, G. Cacciapaglia, H. Cai, T. Flacke, A. Parolini and H. Serôdio, Singlets in composite Higgs models in light of the LHC $750 \mathrm{GeV}$ diphoton excess, Phys. Rev. D 94 (2016) 015004 [arXiv: 1512.07242] [INSPIRE].

[10] M. Chala, G. Nardini and I. Sobolev, Unified explanation for dark matter and electroweak baryogenesis with direct detection and gravitational wave signatures, Phys. Rev. D 94 (2016) 055006 [arXiv: 1605.08663] [INSPIRE].

[11] B. Gripaios, M. Nardecchia and T. You, On the Structure of Anomalous Composite Higgs Models, Eur. Phys. J. C 77 (2017) 28 [arXiv:1605.09647] [INSPIRE]. 
[12] M. Chala, R. Gröber and M. Spannowsky, Searches for vector-like quarks at future colliders and implications for composite Higgs models with dark matter, JHEP 03 (2018) 040 [arXiv: 1801.06537] [INSPIRE].

[13] M. Chala, R. Gröber and M. Spannowsky, Interplay between collider searches for vector-like quarks and dark matter searches in composite Higgs models, Int. J. Mod. Phys. A 34 (2019) 1940011 [INSPIRE].

[14] G. Cacciapaglia, G. Ferretti, T. Flacke and H. Serôdio, Light scalars in composite Higgs models, Front. in Phys. 7 (2019) 22 [arXiv:1902.06890] [INSPIRE].

[15] M. Ramos, Composite dark matter phenomenology in the presence of lighter degrees of freedom, JHEP 07 (2020) 128 [arXiv:1912.11061] [INSPIRE].

[16] J.R. Espinosa, B. Gripaios, T. Konstandin and F. Riva, Electroweak Baryogenesis in Non-minimal Composite Higgs Models, JCAP 01 (2012) 012 [arXiv:1110.2876] [INSPIRE].

[17] V. Vaskonen, Electroweak baryogenesis and gravitational waves from a real scalar singlet, Phys. Rev. D 95 (2017) 123515 [arXiv:1611.02073] [InSPIRE].

[18] A. Beniwal, M. Lewicki, J.D. Wells, M. White and A.G. Williams, Gravitational wave, collider and dark matter signals from a scalar singlet electroweak baryogenesis, JHEP 08 (2017) 108 [arXiv: 1702.06124] [INSPIRE].

[19] Z. Kang, P. Ko and T. Matsui, Strong first order EWPT $\& 3$ strong gravitational waves in $Z_{3}$-symmetric singlet scalar extension, JHEP 02 (2018) 115 [arXiv:1706. 09721] [INSPIRE].

[20] B. Grzadkowski and D. Huang, Spontaneous CP-Violating Electroweak Baryogenesis and Dark Matter from a Complex Singlet Scalar, JHEP 08 (2018) 135 [arXiv:1807.06987] [INSPIRE].

[21] S. De Curtis, L. Delle Rose and G. Panico, Composite Dynamics in the Early Universe, JHEP 12 (2019) 149 [arXiv: 1909.07894] [INSPIRE].

[22] J. Liu, C.E.M. Wagner and X.-P. Wang, A light complex scalar for the electron and muon anomalous magnetic moments, JHEP 03 (2019) 008 [arXiv:1810.11028] [INSPIRE].

[23] N. Craig, H.K. Lou, M. McCullough and A. Thalapillil, The Higgs Portal Above Threshold, JHEP 02 (2016) 127 [arXiv: 1412.0258] [INSPIRE].

[24] M. Ruhdorfer, E. Salvioni and A. Weiler, A Global View of the Off-Shell Higgs Portal, SciPost Phys. 8 (2020) 027 [arXiv: 1910.04170] [INSPIRE].

[25] C.A. Baker et al., An Improved experimental limit on the electric dipole moment of the neutron, Phys. Rev. Lett. 97 (2006) 131801 [hep-ex/0602020] [INSPIRE].

[26] ACME collaboration, Improved limit on the electric dipole moment of the electron, Nature 562 (2018) 355 [INSPIRE].

[27] R. Franceschini et al., Digamma, what next?, JHEP 07 (2016) 150 [arXiv:1604.06446] [INSPIRE].

[28] B. Gripaios and D. Sutherland, An operator basis for the Standard Model with an added scalar singlet, JHEP 08 (2016) 103 [arXiv: 1604.07365] [INSPIRE].

[29] S. Banerjee, M. Chala and M. Spannowsky, Top quark FCNCs in extended Higgs sectors, Eur. Phys. J. C 78 (2018) 683 [arXiv:1806.02836] [InSPIRE].

[30] J.A. Aguilar-Saavedra and G.C. Branco, Probing top flavor changing neutral scalar couplings at the CERN LHC, Phys. Lett. B 495 (2000) 347 [hep-ph/0004190] [INSPIRE]. 
[31] D. Atwood, S.K. Gupta and A. Soni, Constraining the flavor changing Higgs couplings to the top-quark at the LHC, JHEP 10 (2014) 057 [arXiv:1305.2427] [INSPIRE].

[32] A. Papaefstathiou and G. Tetlalmatzi-Xolocotzi, Rare top quark decays at a $100 \mathrm{TeV}$ proton-proton collider: $t \rightarrow b W Z$ and $t \rightarrow h c$, Eur. Phys. J. C 78 (2018) 214 [arXiv: 1712.06332] [INSPIRE].

[33] ATLAS collaboration, Search for top quark decays $t \rightarrow q H$, with $H \rightarrow \gamma \gamma$, in $\sqrt{s}=13 \mathrm{TeV}$ pp collisions using the ATLAS detector, JHEP 10 (2017) 129 [arXiv:1707.01404] [INSPIRE].

[34] ATLAS collaboration, Search for flavor-changing neutral currents in top quark decays $t \rightarrow H c$ and $t \rightarrow H u$ in multilepton final states in proton-proton collisions at $\sqrt{s}=13 \mathrm{TeV}$ with the ATLAS detector, Phys. Rev. D 98 (2018) 032002 [arXiv: 1805.03483] [InSPIRE].

[35] M. Chala, J. Santiago and M. Spannowsky, Constraining four-fermion operators using rare top decays, JHEP 04 (2019) 014 [arXiv: 1809.09624] [INSPIRE].

[36] M. Barros et al., Study of interference effects in the search for flavour-changing neutral current interactions involving the top quark and a photon or a $Z$ boson at the LHC, Eur. Phys. J. Plus 135 (2020) 339 [arXiv:1909.08443] [InSPIRE].

[37] CMS collaboration, Search for Higgs boson off-shell production in proton-proton collisions at 7 and $8 \mathrm{TeV}$ and derivation of constraints on its total decay width, JHEP 09 (2016) 051 [arXiv: 1605.02329] [INSPIRE].

[38] CMS collaboration, Search for low-mass resonances decaying into bottom quark-antiquark pairs in proton-proton collisions at $\sqrt{s}=13$ TeV, Phys. Rev. D 99 (2019) 012005 [arXiv: 1810.11822] [INSPIRE].

[39] CMS collaboration, Search for high mass dijet resonances with a new background prediction method in proton-proton collisions at $\sqrt{s}=13 \mathrm{TeV}$, JHEP 05 (2020) 033 [arXiv: 1911.03947] [INSPIRE].

[40] R. Harnik, J. Kopp and J. Zupan, Flavor Violating Higgs Decays, JHEP 03 (2013) 026 [arXiv: 1209.1397] [INSPIRE].

[41] UTFIT collaboration, Model-independent constraints on $\Delta F=2$ operators and the scale of new physics, JHEP 03 (2008) 049 [arXiv:0707.0636] [INSPIRE].

[42] Top Quark Working Group collaboration, Working Group Report: Top Quark, in Community Summer Study 2013: Snowmass on the Mississippi, 11, 2013 [arXiv:1311.2028] [INSPIRE].

[43] Particle Data Group collaboration, Review of Particle Physics, Phys. Rev. D 98 (2018) 030001 [INSPIRE].

[44] D.B. Kaplan and H. Georgi, SU(2) $\times$ U(1) Breaking by Vacuum Misalignment, Phys. Lett. B 136 (1984) 183 [inSPIRE].

[45] D.B. Kaplan, H. Georgi and S. Dimopoulos, Composite Higgs Scalars, Phys. Lett. B 136 (1984) 187 [INSPIRE].

[46] ATLAS collaboration, Search for flavour-changing neutral current top-quark decays $t \rightarrow q Z$ in proton-proton collisions at $\sqrt{s}=13 \mathrm{TeV}$ with the ATLAS detector, JHEP 07 (2018) 176 [arXiv: 1803.09923] [INSPIRE].

[47] R. Brun and F. Rademakers, ROOT: An object oriented data analysis framework, Nucl. Instrum. Meth. A 389 (1997) 81 [INSPIRE]. 
[48] M. Cacciari, G.P. Salam and G. Soyez, FastJet User Manual, Eur. Phys. J. C 72 (2012) 1896 [arXiv: 1111.6097] [INSPIRE].

[49] J. Alwall et al., The automated computation of tree-level and next-to-leading order differential cross sections, and their matching to parton shower simulations, JHEP 07 (2014) 079 [arXiv: 1405.0301] [INSPIRE].

[50] T. Sjöstrand et al., An introduction to PYTHIA 8.2, Comput. Phys. Commun. 191 (2015) 159 [arXiv: 1410.3012] [INSPIRE].

[51] M. Czakon and A. Mitov, Top++: A Program for the Calculation of the Top-Pair Cross-Section at Hadron Colliders, Comput. Phys. Commun. 185 (2014) 2930 [arXiv:1112.5675] [INSPIRE].

[52] ATLAS collaboration, Search for supersymmetry in events with four or more leptons in $\sqrt{s}=13$ TeV pp collisions with ATLAS, Phys. Rev. D 98 (2018) 032009 [arXiv:1804.03602] [INSPIRE].

[53] L3 collaboration, A Study of four fermion processes at LEP, Phys. Lett. B 321 (1994) 283 [INSPIRE].

[54] A. Alloul, N.D. Christensen, C. Degrande, C. Duhr and B. Fuks, FeynRules 2.0 - A complete toolbox for tree-level phenomenology, Comput. Phys. Commun. 185 (2014) 2250 [arXiv: 1310.1921] [INSPIRE].

[55] R.D. Ball et al., Parton distributions with LHC data, Nucl. Phys. B 867 (2013) 244 [arXiv: 1207.1303] [INSPIRE].

[56] DELPHES 3 collaboration, DELPHES 3, A modular framework for fast simulation of a generic collider experiment, JHEP 02 (2014) 057 [arXiv: 1307.6346] [INSPIRE].

[57] M. Cacciari, G.P. Salam and G. Soyez, The anti- $k_{t}$ jet clustering algorithm, JHEP 04 (2008) 063 [arXiv: 0802.1189] [INSPIRE].

[58] A.L. Read, Presentation of search results: The $C L_{s}$ technique, J. Phys. G 28 (2002) 2693 [INSPIRE].

[59] E. Busato, D. Calvet and T. Theveneaux-Pelzer, OpTHyLiC: an Optimised Tool for Hybrid Limits Computation, Comput. Phys. Commun. 226 (2018) 136 [arXiv:1502.02610] [INSPIRE]. 